\title{
Some Solvable Models of Shear Dispersion
}

Uwe Mikolajewicz

UDC 551.465.15

\section{Summary}

A new method is introduced to solve the turbulent diffusion equation with depth-dependent current. Some simple models are derived from it, which especially discuss the influence of nonlinear current profiles, boundaries and depth-dependence of the exchange coefficients.

\section{Einige lösbare Scherdispersionsmodelle (Zusammenfassung)}

Eine neue Methode zur Lösung der turbulenten Diffusionsgleichung mit tiefenabhängiger Geschwindigkeit wird entwickelt. Damit werden einige einfache Modelle aufgestellt, die besonders den Einfluß von nichtlinearen Stromprofilen, undurchdringlichen Wänden und Tiefenabhängigkeit der Austauschkoeffizienten darstellen.

\section{Sur quelques modèles resolubles de la dispersion de cisaillement (Résumé)}

On présente une nouvelle méthode pour résoudre l'équation de la diffusion de la turbulence avec un courant dépendant de la profondeur. Quelques modèles simples en dérivent, qui représentent surtout l'influence de profils de courants non linéaires, des limites imperméables et de la dépendance à la profondeur des coefficients d'échange.

\section{List of symbols}

$\begin{array}{ll}A_{n j} & \text { exchange coefficient } \\ A_{X v}, A_{Y v}, A_{Z v} & \text { element of power series expansion of } A_{11}, A_{22} \text { or } A_{33} \\ A_{1}, A_{3} & \text { maximum of } A_{11}, A_{33} \\ A_{X \text { eff }} & \text { effective exchange coefficient (for definition see section 4.2) } \\ a_{v} & \text { coefficient of power series expansion of velocity } u \\ b_{v} & \text { coefficient of power series expansion of the velocity } \\ C & \text { calibrated concentration } \\ C_{N} & \text { approximated calibrated concentration } \\ c_{v} & \text { discrete Fourier transform coefficient of the velocity gradient } \\ f & \text { function (see Appendix 1) } \\ g & \text { coefficient of the power series expansion of } f \\ H & \text { depth of the channel } \\ h & \text { half the depth of the channel } \\ \mathrm{i} & \text { V-1 } \\ j & \text { index for space co-ordinates }(j=1,2,3) \\ K & \text { maximum of the vertical exchange coefficient (section } 4.2) \\ k, l, m & \text { indices giving the degree of the moments with respect to } x, y, z \\ M & \text { total mass } \\ N, N_{X}, N_{Y}, N_{Z}, N_{\alpha} & \text { boundaries of summation } \\ N_{\mathrm{BV}} & \text { Brunt-Väisälä frequency } \\ n & \text { index for space co-ordinate }(n=1,2,3) \\ P, Q & \text { dimensionless functions (see section 3.4) } \\ p, q, r & \text { summation indices (see Appendix 1) } \\ R_{\mathrm{L}} & \text { Lagrangian correlation funetion } \\ & \end{array}$




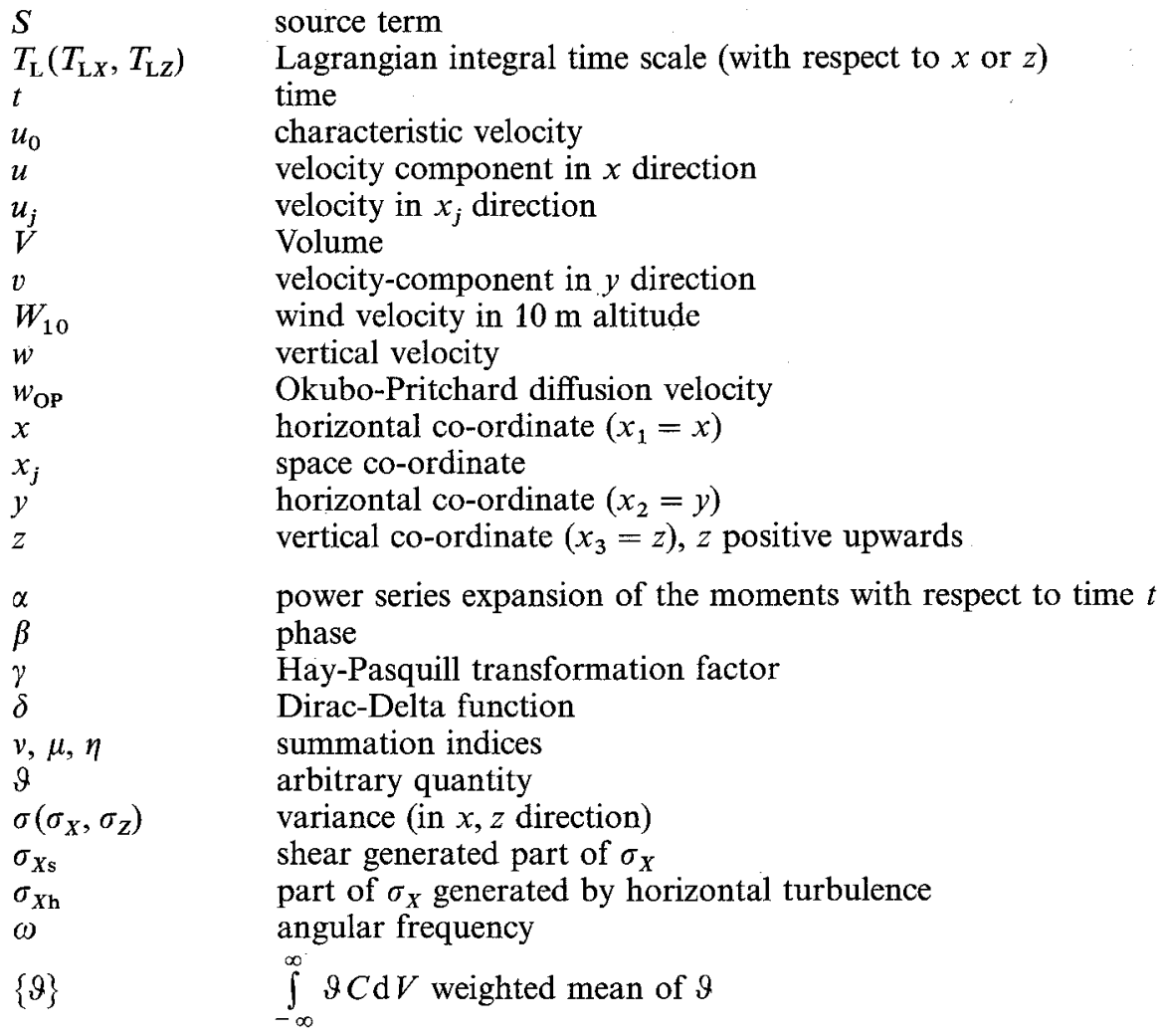

\section{Introduction}

In the discussion of the consequences of environmental pollution on the marine ecosystem it is an important factor to know the spread of the pollutant. The mixing process is often dominated by the effect of shear dispersion (Kullenberg [1974]).

Basic work on modelling this effect has been carried out by Taylor [1953], Bowden [1965], Okubo [1967], Fennel [1979] and Young, Rhines and Garrett [1982], who all consider the case of a momentaneous source.

In the following sections, a new method of solving the shear dispersion problem is introduced and some simple models, describing various basic aspects, are derived from it.

\section{The method}

In this section, a method is developed, that reduces the turbulent diffusion equation with depth-dependent current to a closed system of linear ordinary differential equations for the moments.

The semi-empirical turbulent diffusion equation is

$$
\frac{\partial C}{\partial t}+u_{j} \frac{\partial C}{\partial x_{j}}-\frac{\partial}{\partial x_{n}}\left(A_{n j} \frac{\partial C}{\partial x_{j}}\right)=S\left(x_{j}\right) \delta\left(t-t_{0}\right) .
$$

$t$ is the time, $u_{j}$ is the velocity component in the $x_{j}$-direction, $A_{n j}$ is the exchange coefficient tensor, $S$ is the source term, $\delta$ is the Dirac-Delta function, $n$ and $j$ are indices running from 1 to 3 , and $C$ is the concentration, which is calibrated in such a way, that

$$
\int_{-\infty}^{\infty} C \mathrm{~d} V=1
$$


The weighted mean

$$
\int_{-\infty}^{\infty} \vartheta C \mathrm{~d} V \text { is noted as }\{\vartheta\}
$$

By means of the Reynolds transport theorem

$$
\frac{\mathrm{d}}{\mathrm{d} t}\{\vartheta\}=\int_{V}\left[\frac{\partial}{\partial t}(\vartheta C)+\frac{\partial}{\partial x_{j}}\left(\vartheta C u_{j}\right)\right] \mathrm{d} V
$$

where $\vartheta$ is an arbitrary quantity, and under assumption that both the concentration and its gradient tend to 0 if the distance from the source point tends to infinity, Franz [1982; 1984] obtains from

$$
\frac{\partial}{\partial x_{j}} u_{j}=0
$$

and from, (1) and (2)

$$
\frac{\mathrm{d}}{\mathrm{d} t}\{\vartheta\}=\left\{\frac{\partial \vartheta}{\partial t}+u_{j} \frac{\partial}{\partial x_{j}} \vartheta\right\}+\left\{\frac{\partial}{\partial x_{j}}\left(A_{n j} \frac{\partial \vartheta}{\partial x_{n}}\right)\right\} .
$$

$A_{n j}$ is assumed as usually, 0 for $n \neq j$. By introducing horizontal homogeneity, one can express $A_{n j}$ and $u_{j}$ as power series of the vertical coordinate $z$ :

$$
\begin{gathered}
u(z, t)=u_{1}(z, t)=\sum_{v=0}^{N} a_{v}(t) z^{v} \\
v(z, t)=u_{2}(z, t)=\sum_{v=0}^{N} b_{v}(t) z^{v} \\
A_{11}(z, t)=\sum_{v=0}^{N_{X}} A_{X v} z^{v} \\
A_{22}(z, t)=\sum_{v=0}^{N_{Y}} A_{Y v} z^{v} \\
A_{33}(z, t)=\sum_{v=0}^{N_{Z}} A_{Z v} z^{v} .
\end{gathered}
$$

Setting $\vartheta=x^{k} y^{l} z^{m},(3)$ and (4) result in the reduction formula:

$$
\begin{aligned}
\frac{\mathrm{d}}{\mathrm{d} t}\left\{x^{k} y^{l} z^{m}\right\}= & k \sum_{\nu=0}^{N} a_{\nu}\left\{x^{k-1} y^{l} z^{m+v}\right\} \\
& +l \sum_{v=0}^{N^{\prime}} b_{v}\left\{x^{k} y^{l-1} z^{m+v}\right\} \\
& +k(k-1) \sum_{v=0}^{N_{X}} A_{X \nu}\left\{x^{k-2} y^{l} z^{m+v}\right\} \\
& +l(l-1) \sum_{\nu=0}^{N_{X}} A_{Y v}\left\{x^{k} y^{l-2} z^{m+\nu}\right\} \\
& +m \sum_{\nu=0}^{N_{Z}}(m+v-1) A_{Z v}\left\{x^{k} y^{l} z^{m+v-2}\right\}
\end{aligned}
$$


If $N_{Z} \leqq 2$, a closed system of linear differential equations is described, which may be solved successively. In this way, it is possible to calculate the exact solution for any desired moment of the concentration distribution, which can inversely be calculated from its moments. A method, allowing the calculation of an approximate distribution when only a limited number of moments are known, is given in Appendix 1.

The solution for some simple cases are computed in the following.

\section{Space-independent exchange coefficient}

In this section, the exchange coefficient is assumed to be space-independent, and the distribution shall not be affected by any boundaries.

\subsection{Steady current}

To shorten the formulas this case will be first discussed in two dimensions, the $x$-axis lies in the direction of the current and $z$ is positive upwards. Thus (5) reduces to:

$$
\begin{aligned}
\frac{\mathrm{d}}{\mathrm{d} t}\left\{x^{k} z^{m}\right\}= & k \sum_{v=0}^{N} a_{v}\left\{x^{k-1} z^{m+v}\right\} \\
& +k(k-1) A_{X 0}\left\{x^{k-2} z^{m}\right\} \\
& +m(m-1) A_{Z 0}\left\{x^{k} z^{m-2}\right\}
\end{aligned}
$$

This describes a set of ordinary linear differential equations, which can be solved successively by time integration.

To obtain the variance with $N=3, t_{0}=0$ and

$$
S\left(x_{\mathrm{i}}\right)=\delta(x) \delta(y) \delta(z)
$$

the following moments have to be calculated:

$$
\begin{aligned}
\{z\}= & 0 \\
\left\{z^{2}\right\}= & 2 A_{Z 0} t \\
\left\{z^{3}\right\}= & 0 \\
\left\{z^{4}\right\}= & 12 A_{Z 0}^{2} t^{2} \\
\left\{z^{5}\right\}= & 0 \\
\left\{z^{6}\right\}= & 120 A_{Z 0}^{3} t^{3} \\
\{x\}= & a_{0} t+a_{2} A_{Z 0} t^{2} \\
\{x z\}= & a_{1} A_{Z 0} t^{2}+4 a_{3} A_{Z 0}^{2} t^{3} \\
\left\{x z^{2}\right\}= & 2 a_{0} A_{Z 0} t^{2}+\frac{14}{3} a_{2} A_{Z 0}^{2} t^{3} \\
\left\{x z^{3}\right\}= & 6 a_{1} A_{Z 0}^{2} t^{3}+36 a_{3} A_{Z 0}^{3} t^{4} \\
\left\{x^{2}\right\}= & 2 A_{X 0} t+a_{0}^{2} t^{2}+\left(\frac{2}{3} a_{1}^{2}+2 a_{0} a_{2}\right) A_{Z 0} t^{3} \\
& +\left(\frac{7}{3} a_{2}^{2}+5 a_{1} a_{3}\right) A_{Z 0}^{2} t^{4}+\frac{72}{5} a_{3}^{2} A_{Z 0}^{3} t^{5} .
\end{aligned}
$$

Thus, for the variance $\sigma_{X}^{2}=\left\{x^{2}\right\}-\{x\}^{2}$, this yields:

$$
\sigma_{X}^{2}=2 A_{X 0} t+\frac{2}{3} a_{1}^{2} A_{Z 0} t^{3}+\left(\frac{4}{3} a_{2}^{2}+5 a_{1} a_{3}\right) A_{Z 0}^{2} t^{4}+\frac{72}{5} a_{3}^{2} A_{Z 0}^{3} t^{5} .
$$


If the distribution can be described by a $\delta$-function at $t=0$, then the odd moments in the $z$ direction are always equal to 0 . The even moments are the same as those resulting from Fickian diffusion without a current profile.

The vertical moments in this case are not affected by the current profile at all, and are expressed by:

$$
\begin{aligned}
\left\{z^{2 \eta+1}\right\} & =0 \\
\left\{z^{2 \eta}\right\} & =\frac{(2 \eta) !}{\eta !} A_{Z 0}^{\eta} t^{\eta} .
\end{aligned}
$$

For the linear current profile, the variance reduces to:

$$
\sigma_{X}^{2}=2 A_{X 0} t+\frac{2}{3} A_{Z 0} a_{1}^{2} t^{3}
$$

which was first given by Okubo [1967]. He obtained this result by Laplace transformation of the turbulent diffusion equation, and soluting the resulting linear partial differential equation of first order with the method of characteristics.

In many experiments, an increase of the variance proportional to $t^{3}$ was found, e.g. by Okubo [1971] and Kullen berg [1974], which leads to the assumption that - in those cases - the linear shear is dominating. In the following, the effect of the nonlinearity will be compared with that term. A term will be considered as significant if it is greater than $5 \%$ of the linear shear dispersion term.

$$
\begin{aligned}
& 2 A_{X 0} t \text { is significant, if } t<8 \sqrt{A_{X 0} /\left(A_{Z 0} a_{1}^{2}\right)} \\
& a_{2} \quad \text { is significant, if } A_{Z 0} t>a_{1}^{2} /\left(40 a_{2}^{2}\right) \\
& a_{3} \text { is significant, if } A_{Z 0} t>a_{1} /\left(150 a_{3}\right)
\end{aligned}
$$

The influence of the nonlinearities grows with time, and dominates for large diffusion times.

To find out whether or not the resulting distribution is Gaussian, the 3rd and 4th moments are calculated. The result, if assuming $a_{0}=0$ for simplicity, is:

$$
\begin{aligned}
\left\{x^{2} z\right\}= & 5 a_{1} a_{2} A_{Z 0}^{2} t^{4}+32 a_{2} a_{3} A_{Z 0}^{3} t^{5} \\
\left\{x^{2} z^{2}\right\}= & 4 A_{X 0} t^{2}+\frac{10}{3} a_{1}^{2} A_{Z 0}^{2} t^{4}+\frac{278}{15} a_{2}^{2} A_{Z 0}^{3} t^{5} \\
& +\frac{202}{5} a_{1} a_{3} A_{Z 0}^{3} t^{5}+\frac{824}{5} a_{3}^{2} A_{Z 0}^{4} t^{6} \\
\left\{x^{3}\right\}= & 6 a_{2} A_{X 0} A_{Z 0} t^{3}+5 a_{1}^{2} a_{2} A_{Z 0}^{2} t^{5} \\
& +\left(60 a_{1} a_{2} a_{3}+\frac{139}{15} a_{2}^{3}\right) A_{Z 0}^{3} t^{6}+\frac{8352}{35} a_{2} a_{3}^{2} A_{Z 0}^{4} t^{7} \\
\left\{x^{3} z\right\}= & 6 a_{1} A_{X 0} A_{Z 0} t^{3}+24 a_{3} A_{X 0} A_{Z 0}^{2} t^{4}+2 a_{1}^{3} A_{Z 0}^{2} t^{5} \\
& +\left(\frac{496}{15} a_{1} a_{2}^{2}+\frac{181}{5} a_{1}^{2} a_{3}\right) A_{Z 0}^{3} t^{6} \\
& +\left(\frac{9436}{35} a_{2}^{2} a_{3}+\frac{10284}{35} a_{1} a_{3}^{2}\right) A_{Z 0}^{4} t^{7}+\frac{35208}{35} a_{3}^{3} A_{Z 0}^{5} t^{8}
\end{aligned}
$$




$$
\begin{aligned}
\left\{x^{4}\right\}= & 12 A_{X 0}^{2} t^{2}+8 a_{1}^{2} A_{X 0} A_{Z 0} t^{4}+\left(\frac{136}{5} a_{2}^{2}+60 a_{1} a_{3}\right) A_{X 0} A_{Z 0}^{2} t^{5} \\
& +\frac{864}{5} a_{3}^{2} A_{X 0} A_{Z 0}^{3} t^{6}+\frac{4}{3} a_{1}^{4} A_{Z 0}^{2} t^{6} \\
& +\left(\frac{1084}{35} a_{1}^{3} a_{3}+\frac{4472}{105} a_{1}^{2} a_{2}^{2}\right) A_{Z 0}^{3} t^{7} \\
& +\left(\frac{12891}{35} a_{1}^{2} a_{3}^{2}+\frac{5473}{105} a_{2}^{4}+\frac{7632}{7} a_{1} a_{2}^{2} a_{3}\right) A_{Z 0}^{4} t^{8} \\
& +\left(\frac{86944}{35} a_{1} a_{3}^{3}+\frac{51392}{15} a_{2}^{2} a_{3}^{2}\right) A_{Z 0}^{5} t^{9} \\
& +\frac{1340084}{175} a_{3}^{4} A_{Z 0}^{6} t^{10} .
\end{aligned}
$$

Referred to the centre of gravity, this yields:

$$
\begin{aligned}
\left\{x_{0}^{3}\right\}= & \left\{x^{3}\right\}-3\{x\}\left\{x^{2}\right\}+2\{x\}^{3} \\
= & 3 a_{1}^{2} a_{2} A_{Z 0}^{2} t^{5}+\left(45 a_{1} a_{2} a_{3}+\frac{64}{15} a_{2}^{3}\right) A_{Z 0}^{3} t^{6} \\
& +\frac{1368}{7} a_{2} a_{3}^{2} A_{Z 0}^{4} t^{7} \\
\left\{x_{0}^{4}\right\}= & \left\{x^{4}\right\}-4\left(x^{3}\right\}\{x\}+6\left\{x^{2}\right\}\{x\}^{2}-3\{x\}^{4} \\
= & 12 A_{X 0}^{2} t^{2}+8 a_{1}^{2} A_{X 0} A_{Z 0} t^{4}+\left(\frac{76}{5} a_{2}^{2}+60 a_{1} a_{3}\right) A_{X 0} A_{Z 0}^{2} t^{5} \\
& +\frac{864}{5} a_{3}^{2} A_{X 0} A_{Z 0}^{3} t^{6}+\frac{4}{3} a_{1}^{4} A_{Z 0}^{2} t^{6} \\
& +\left(\frac{1084}{35} a_{1}^{3} a_{3}-\frac{2792}{105} a_{1}^{2} a_{2}^{2}\right) A_{Z 0}^{3} t^{7} \\
& +\left(\frac{12891}{35} a_{1}^{2} a_{3}^{2}+\frac{912}{35} a_{2}^{4}+\frac{6162}{7} a_{1} a_{2}^{2} a_{3}\right) A_{Z 0}^{4} t^{8} \\
& +\left(\frac{86944}{35} a_{1} a_{3}^{3}+\frac{268592}{105} a_{2}^{2} a_{3}^{2}\right) A_{Z 0}^{5} t^{9}+\frac{1340084}{175} a_{3}^{4} A_{Z 0}^{6} t^{10} .
\end{aligned}
$$

The 3 rd moment, which gives the skewness of the distribution, is only different from 0 , if $a_{2}$ is different from 0 . The skewness of the distribution is produced by the part of the current that is symmetric to $z=0$.

$$
\begin{aligned}
\left\{x_{0}^{4}\right\}-3 \sigma_{X}^{4}= & -\frac{4}{5} a_{2}^{2} A_{X 0} A_{Z 0}^{2} t^{5}+\left(\frac{384}{35} a_{1}^{3} a_{3}-\frac{3352}{105} a_{1}^{2} a_{2}^{2}\right) A_{Z 0}^{3} t^{7} \\
& +\left(\frac{1650}{7} a_{1}^{2} a_{3}^{2}+\frac{2176}{105} a_{2}^{4}+\frac{5882}{7} a_{1} a_{2}^{2} a_{3}\right) A_{Z 0}^{4} t^{8} \\
& +\left(\frac{71824}{35} a_{1} a_{3}^{3}+\frac{256496}{105} a_{2}^{2} a_{3}^{2}\right) A_{Z 0}^{5} t^{9} \\
& +\frac{246244}{35} a_{3}^{4} A_{Z 0}^{6} t^{10}
\end{aligned}
$$


Fore a more convenient representation, the 4 th moment, as resulting from a Gaussian distribution with the same variance, has been subtracted from (10). The linear part only contributes to the deviation from the Gaussian distribution when interacting with the nonlinear parts of the current profile; a result to be expected, because Okubo [1967] and Carter and Okubo [1965] have shown that the solution in the case of a linear current profile is Gaussian.

An algorithm that may help in calculating higher moments is given below. With

$$
\left\{x^{k} z^{m}\right\}=\sum_{\mu=0}^{N_{\alpha}} \alpha(k, m, \mu) t^{\mu}
$$

$\left(5^{\mathrm{I}}\right)$ results in:

$$
\begin{aligned}
\alpha(k, m, \mu)= & \frac{k}{\mu} \sum_{\nu=0}^{N} a_{v} \alpha(k-1, m+v, \mu-1) \\
& +\frac{k(k-1)}{\mu} A_{X 0} \alpha(k-2, m, \mu-1) \\
& +\frac{m(m-1)}{\mu} A_{Z 0} \alpha(k, m-2, \mu-1) .
\end{aligned}
$$

For negative indices $\alpha$ is equal to 0 . The same is valid for $\mu=0$. An exception is

$$
\alpha(0,0,0)=1 \text {. }
$$

$N_{\alpha}$ is less than or equal to $(N+1) k+m / 2$.

Thus it is possible to compute every desired moment of the distribution with a computer, if $a_{v}$ and $A_{j j}$ are known.

\subsection{Oscillating current}

It is assumed that

$$
u(z, t)=\sum_{v=0}^{3} a_{v} z^{v} \sin (\omega t+\beta)
$$

where $\omega$ is the angular frequency and $\beta$ the phase of the motion.

For large diffusion times, $\omega t \gg 1\left(5^{\mathrm{I}}\right)$ in combination with the initial conditions (6) and the current (15) results in the following approximated expression of the variance:

$$
\begin{aligned}
\sigma_{X}^{2}= & 2 A_{X 0} t \\
& +A_{Z 0} \frac{a_{1}^{2}}{\omega^{2}}(2 t+t \cos 2(\omega t+\beta)) \\
& +A_{Z 0}^{2} \frac{a_{2}^{2}}{\omega^{2}}\left(8 t^{2}+4 t^{2} \cos 2(\omega t+\beta)\right) \\
& +A_{Z 0}^{2} \frac{a_{1} a_{3}}{\omega^{2}}\left(18 t^{2}+12 t^{2} \cos 2(\omega t+\beta)\right) \\
& +A_{Z 0}^{3} \frac{a_{3}^{2}}{\omega^{2}}\left(96 t^{3}+60 t^{3} \cos 2(\omega t+\beta)\right) .
\end{aligned}
$$

The complete solution is given in Appendix 2. 


\subsection{Unspecified time dependence of the current}

Another problem arises if one has the records of two current meters at the same location, but different depths $z_{1}$ and $z_{2}$. If $\left|z_{1}-z_{2}\right|$ is not too large, the depth-dependence of the current can be approximated by

$$
\frac{\partial u(z, t)}{\partial z}=\frac{u\left(z_{1}, t\right)-u\left(z_{2}, t\right)}{z_{1}-z_{2}} .
$$

The same can be carried out for the $y$ component. If the source point of the distribution lies between $z_{1}$ and $z_{2}$ and when setting $z=0$ at this point, the current profile is given approximately by:

$$
\begin{aligned}
u(z, t) & \approx u(0, t)+\frac{\partial u(t)}{\partial z} z \\
& =u(0, t)+z \sum_{v=0}^{N} \frac{c_{v}}{2}\left(\mathrm{e}^{\mathrm{i} \beta_{v}} \mathrm{e}^{\mathrm{i} \omega_{v} t}+\mathrm{e}^{-\mathrm{i} \beta_{v}} \mathrm{e}^{-\mathrm{i} \omega_{v} t}\right),
\end{aligned}
$$

where $c_{v}$ is the Fourier transformed of the velocity gradient. Transforming the equation into a co-ordinate system moving with $u(0, t),\left(5^{5}\right)$ yields with $\beta_{0}=0$ and $\omega_{0}=0$ :

$$
\begin{aligned}
& \left\{z^{2}\right\}=2 A_{Z 0} t \\
& \{x\}=0 \\
& \{x z\}=c_{0} A_{Z 0} t^{2} \\
& +A_{Z 0} \sum_{\nu=1}^{N} c_{v}\left[\frac{\mathrm{e}^{\mathrm{i} \beta_{v}}}{-\omega_{v}^{2}}\left(\left(\mathrm{i} \omega_{v} t-1\right) \mathrm{e}^{\mathrm{i} \omega_{\nu} t}+1\right)+\frac{\mathrm{e}^{-\mathrm{i} \beta_{v}}}{-\omega_{v}^{2}}\left(\left(-\mathrm{i} \omega_{v} t-1\right) \mathrm{e}^{-\mathrm{i} \omega_{v} t}+1\right)\right] \\
& \sigma_{X}^{2}=\left\{x^{2}\right\} \\
& =2 A_{X 0} t \\
& +\frac{2}{3} c_{0}^{2} A_{Z 0} t^{3} \\
& +A_{Z 0} \sum_{\nu=1}^{N} \frac{c_{0} c_{v}}{\omega_{v}^{2}}\left[\mathrm{e}^{\mathrm{i} \beta_{v}}\left(-\mathrm{i} \omega_{v} t^{2} \mathrm{e}^{\mathrm{i} \omega_{v} t}-2 t-\frac{2 \mathrm{i}}{\omega_{v}} \mathrm{e}^{\mathrm{i} \omega_{v} t}+\frac{2 \mathrm{i}}{\omega_{v}}\right)\right. \\
& \left.+\mathrm{e}^{-\mathrm{i} \beta_{v}}\left(\mathrm{i} \omega_{v} t^{2} \mathrm{e}^{-\mathrm{i} \omega_{v} t}-2 t+\frac{2 \mathrm{i}}{\omega_{v}} \mathrm{e}^{-\mathrm{i} \omega_{v} t}-\frac{2 \mathrm{i}}{\omega_{v}}\right)\right] \\
& +A_{Z 0} \sum_{v=1}^{N} \frac{c_{v}^{2}}{\omega_{v}^{2}}\left[\mathrm{e}^{2 \mathrm{i} \beta_{v}}\left(-\frac{1}{2} t \mathrm{e}^{2 \mathrm{i} \omega_{v} t}-\frac{3}{4} \frac{\mathrm{i}}{\omega_{v}} \mathrm{e}^{2 \mathrm{i} \omega_{v} t}+\frac{\mathrm{i}}{\omega_{v}} \mathrm{e}^{\mathrm{i} \omega_{v} t}-\frac{\mathrm{i}}{4 \omega_{v}}\right)\right. \\
& +2 t+\frac{i}{\omega_{v}} \cdot\left(\mathrm{e}^{\mathrm{i} \omega_{\nu} t}-\mathrm{e}^{-\mathrm{i} \omega_{\nu} t}\right) \\
& \left.+\mathrm{e}^{-2 \mathrm{i} \beta_{v}}\left(-\frac{1}{2} t \mathrm{e}^{-2 \mathrm{i} \omega_{v} t}+\frac{3}{4} \frac{\mathrm{i}}{\omega_{v}} \mathrm{e}^{-2 \mathrm{i} \omega_{v} t}-\frac{\mathrm{i}}{\omega_{v}} \mathrm{e}^{-\mathrm{i} \omega_{v} t}+\frac{\mathrm{i}}{4 \omega_{v}}\right)\right] \\
& +A_{Z 0} \sum_{\nu=1}^{N} \sum_{\substack{\mu=1 \\
\mu \neq v}}^{N} \frac{c_{v} c_{\mu}}{\omega_{v}^{2}}\left[\mathrm { e } ^ { \mathrm { i } ( \beta _ { v } + \beta _ { \mu } ) } \left(-\frac{\omega_{v}}{\omega_{v}+\omega_{\mu}} t \mathrm{e}^{\mathrm{i}\left(\omega_{v}+\omega_{\mu}\right) t}+\frac{\mathrm{i}}{\omega_{\mu}}\left(\mathrm{e}^{\mathrm{i} \omega_{\mu} t}-1\right)\right.\right. \\
& \left.+\left(\frac{-\mathrm{i}}{\omega_{v}+\omega_{\mu}}-\frac{\mathrm{i} \omega_{v}}{\left(\omega_{v}+\omega_{\mu}\right)^{2}}\right) \cdot\left(\mathrm{e}^{\mathrm{i}\left(\omega_{v}+\omega_{\mu}\right) t}-1\right)\right)
\end{aligned}
$$




$$
\begin{gathered}
+\mathrm{e}^{\mathrm{i}\left(\beta_{\mu}-\beta_{v}\right)}\left(\frac{\omega_{v}}{\omega_{\mu}-\omega_{v}} t \mathrm{e}^{\mathrm{i}\left(\omega_{\mu}-\omega_{v}\right) t}+\frac{\mathrm{i}}{\omega_{\mu}}\left(\mathrm{e}^{\mathrm{i} \omega_{\mu} t}-1\right)\right. \\
\left.+\left(\frac{-\mathrm{i}}{\omega_{\mu}-\omega_{v}}+\frac{\mathrm{i} \omega_{v}}{\left(\omega_{\mu}-\omega_{v}\right)^{2}}\right) \cdot\left(\mathrm{e}^{\mathrm{i}\left(\omega_{\mu}-\omega_{v}\right) t}-1\right)\right) \\
+\mathrm{e}^{\mathrm{i}\left(\beta_{v}-\beta_{\mu}\right)}\left(\frac{-\omega_{v}}{\omega_{v}-\omega_{\mu}} t \mathrm{e}^{\mathrm{i}\left(\omega_{\nu}-\omega_{\mu}\right) t}-\frac{\mathrm{i}}{\omega_{\mu}}\left(\mathrm{e}^{-\mathrm{i} \omega_{\mu} t}-1\right)\right. \\
\left.+\left(\frac{-\mathrm{i}}{\omega_{v}-\omega_{\mu}}-\frac{\mathrm{i} \omega_{v}}{\left(\omega_{v}-\omega_{\mu}\right)^{2}}\right) \cdot\left(\mathrm{e}^{\mathrm{i}\left(\omega_{v}-\omega_{\mu}\right) t}-1\right)\right) \\
+\mathrm{e}^{-\mathrm{i}\left(\beta_{v}+\beta_{\mu}\right)}\left(-\frac{\omega_{v}}{\omega_{v}+\omega_{\mu}} t \mathrm{e}^{-\mathrm{i}\left(\omega_{v}+\omega_{\mu}\right) t}-\frac{\mathrm{i}}{\omega_{\mu}}\left(\mathrm{e}^{-\mathrm{i} \omega_{\mu} t}-1\right)\right. \\
\left.\left.+\left(\frac{\mathrm{i}}{\omega_{v}+\omega_{\mu}}+\frac{\mathrm{i} \omega_{v}}{\left(\omega_{v}+\omega_{\mu}\right)^{2}}\right) \cdot\left(\mathrm{e}^{-\mathrm{i}\left(\omega_{v}+\omega_{\mu}\right) t}-1\right)\right)\right] .
\end{gathered}
$$

When $N=1$ and $\beta_{1}=-\pi / 2$ this is identical to the case of the Okubo [1967] model and gives the same result.

(17) makes it possible to calculate the shear generated part of the variance from current measurements direct, if the vertical extension of the tracer is much smaller than the thickness of the layer.

Nevertheless, it is necessary to know the vertical exchange coefficient. Conversely, it should be possible to calculate $A_{z 0}$ from the current data and the measured variances. Kullenberg [1971 and 1974] gives empirical relations between the environmental conditions and the vertical exchange coefficient. Assuming that the stress does not vary with depth, he gives for near surface layers:

$$
A_{Z 0}=9 \cdot 10^{-8} \frac{W_{10}^{2}}{N_{\mathbf{B V}}^{2}}\left|\frac{\mathrm{d} \overline{\left(u_{1}, u_{2}\right)}}{\mathrm{d} z}\right|
$$

$N_{\mathrm{BV}}$ is the Brunt-Väisälä frequency, and $W_{10}$ is the wind velocity at $10 \mathrm{~m}$ height, which has to be greater than $5 \mathrm{~m} / \mathrm{s}$.

For weak winds, where the turbulence is shear-generated, Kullenberg gives:

$$
A_{Z 0} N_{\mathrm{BV}}^{2}=4.1 \cdot 10^{-4} \overline{u_{j}^{\prime 2}}\left|\frac{\mathrm{d} \overline{\left(u_{1}, u_{2}\right)}}{\mathrm{d} z}\right|
$$

\subsection{Time-dependent vertical exchange coefficient}

If the fact that the extension of the patch may be smaller than the largest eddies is taken into consideration, it is possible to use the Taylor formula [1921]:

$$
\sigma_{X}^{2}(t)=2 \int_{0}^{t} \mathrm{~d} t^{\prime} \int_{0}^{t^{\prime}} \mathrm{d} t^{\prime \prime} \overline{u\left(t^{\prime}\right) \cdot u\left(t^{\prime}-t^{\prime \prime}\right)}
$$

which is originally defined for the relative diffusion of two fluid particles. However, it is assumed here that it is still valid if we are dealing with the transport of a passive tracer. This yields in the following the exchange coefficient:

$$
A_{X 0}(t)=\overline{u_{\mathrm{L}}^{\prime 2}} \int_{0}^{t} \mathrm{~d} \tau R_{\mathrm{L}}(\tau)
$$


$R_{\mathrm{L}}$ is the Lagrangian velocity correlation. The integral

$$
\int_{0}^{\infty} \mathrm{d} \tau R_{\mathrm{L}}(\tau)=T_{\mathrm{L}}
$$

is a constant. $R_{\mathrm{L}}$ can be approximated, as was proposed by Taylor [1921], by:

$$
R_{\mathrm{L}}(\tau)=\exp \left(-\tau / T_{\mathrm{L}}\right)
$$

$T_{\mathrm{L}}$ is the Lagrangian integral time scale.

The same calculations can be made for the vertical exchange coefficient $A_{Z 0}$. These calculations yield the following results:

$$
\begin{aligned}
& A_{X 0}=A_{1}\left(1-\exp \left(-t / T_{\mathrm{L} X}\right)\right. \\
& A_{Z 0}=A_{3}\left(1-\exp \left(-t / T_{\mathrm{L} Z}\right)\right.
\end{aligned}
$$

In combination with a steady linear current profile, and with $\left(5^{\mathrm{I}}\right)$, this yields:

$$
\begin{aligned}
\left\{z^{2}\right\}= & 2 A_{3} t\left[1-\frac{T_{\mathrm{L} Z}}{t}\left(1-\exp \left(-t / T_{\mathrm{LZ}}\right)\right)\right] \\
\{x z\}= & 2 A_{3} a_{1}\left[\frac{t^{2}}{2}-t T_{\mathrm{L} Z}-T_{\mathrm{L} Z}^{2}\left(\exp \left(-t / T_{\mathrm{L} Z}\right)-1\right)\right] \\
\left\{x^{2}\right\}= & \sigma_{X}^{2} \\
= & \frac{2}{3} A_{3} a_{1}^{2} t^{3}\left[1-3 \frac{T_{\mathrm{L} Z}}{t}+6\left(\frac{T_{\mathrm{L} Z}}{t}\right)^{2}\right. \\
& \left.-6\left(\frac{T_{\mathrm{L} Z}}{t}\right)^{3} \cdot\left(1-\exp \left(-\frac{t}{T_{\mathrm{L} Z}}\right)\right)\right] \\
& +2 A_{1} t\left[1-\frac{T_{\mathrm{L} X}}{t}\left(1-\exp \left(-\frac{t}{T_{\mathrm{L} X}}\right)\right)\right] \\
= & \frac{2}{3} A_{3} a_{1}^{2} t^{3} P(t)+2 A_{1} t Q(t) .
\end{aligned}
$$

The dependence of $P(t)$ and $Q(t)$ on time is shown in Fig.1. For diffusion times much smaller than the Lagrangian integral time scale, $Q$ can be approximated by the function $t / 2 T_{\mathbf{L X}}$. For larger times, $Q=1$ is a good approximation.

Fig. 2 gives the relationship between nondimensional time $t / T_{\mathrm{L} Z}$ and the contribution of the horizontal turbulent diffusion to the variance $\sigma_{X \mathrm{~h}}^{2}$ :

$$
\sigma_{X \mathrm{~h}}^{2}=2 A_{1} t\left[1-\frac{T_{\mathrm{L} X}}{t}\left(1-\exp \left(-t / T_{\mathrm{LX}}\right)\right)\right] \text {. }
$$

This expression was first given by Taylor [1921]. For small times the variance increases proportional to $t^{2}$, resulting in the Okubo-Pritchard model (Okubo [1962]) if horizontal isotropy is assumed and the model is expanded in 3 dimensions. The Okubo-Pritchard diffusion velocity $w_{\mathrm{OP}}$ is given by:

$$
w_{\mathrm{OP}}=\sqrt{\frac{A_{1}}{T_{\mathrm{LX}}}}=\sqrt{u^{\prime 2}} .
$$

For times much larger than $T_{\mathrm{LX}}$, the present model yields Fickian diffusion with $A_{1}$. The characteristic time scale $t_{\mathrm{c}}$ for the distinction between the two cases is $t_{\mathrm{c}}=2 T_{\mathrm{LX}}$. 


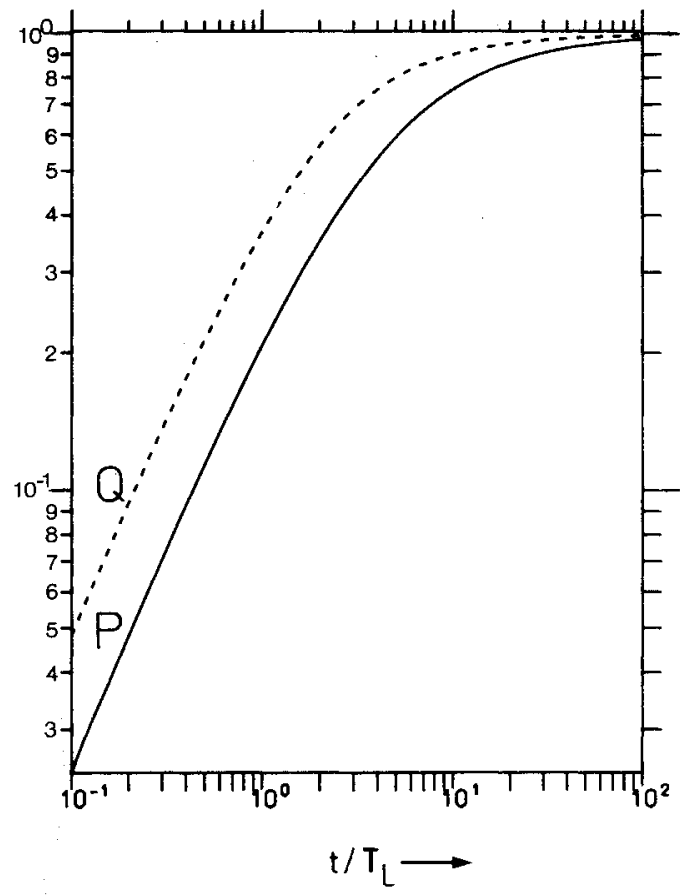

Fig. 1. $\quad P$ and $Q$ versus time $t / T_{\mathbf{L}}$ (for definitions see section 3.4 equation (19))

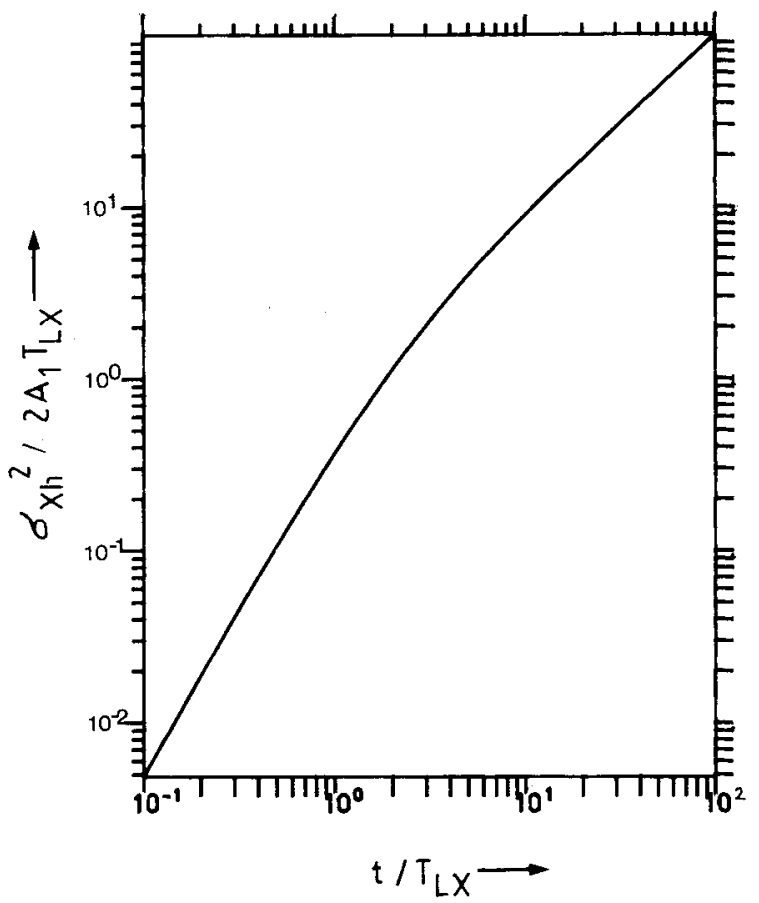

Fig. 2. Variance generated by horizontal turbulent diffusion $\sigma_{X \mathrm{~h}}^{2} / 2 A_{1} T_{\mathrm{LX}}$ versus time $t / T_{\mathrm{L} X}$ (for definitions see section 3.4) 
Neumann [1978] applied this model to atmospheric diffusion, obtaining quite good results. It may be possible to apply this model to marine turbulent diffusion too. Results from large-scale experiments with drifters in the Pacific, carried out by Kirwan et al. [1978], show the same characteristics as Fig. 2. $T_{\mathrm{L} X}$ values of between 4 and 10 days are estimated from the graphs.

For small diffusion times, as defined by $t / T_{\mathrm{LZ}} \ll 1, P(t)$ can be approximated by $P(t)$ $=t / 4 T_{\mathrm{L} Z}$, whereas $P(t)$ is approximately 1 for large times. The shear generated part of the variance $\sigma_{X \mathrm{~s}}^{2}$ increases as $t^{4}$ for small times, whereas for large times $\sigma_{X \mathrm{~s}}^{2}$ increases as $t^{3}$, and the present model becomes equivalent to $\mathrm{Okubo}$ 's [1977]. The relationship between $\sigma_{X \mathrm{~s}}^{2}$ and non-dimensional time $t / T_{\mathrm{L} Z}$ is shown in Fig. 3 .

$A_{1}$ and $T_{\mathrm{LX}}$ can be obtained from tracer experiments. $A_{1}$ is given by:

$$
A_{1}=\overline{u_{\mathrm{L}}^{\prime 2}} T_{\mathrm{L} X}
$$

$u_{\mathrm{L}}^{\prime}$ must be measured in a Lagrangian frame of reference. Under certain conditions (stationarity and homogeneity) this is equal to the velocities $\overline{u^{\prime 2}}$ measured in an Eulerian frame. Thus, there are various ways of determining $A_{1}, T_{\mathrm{LX}}$ and $\overline{u^{\prime 2}}$ from simultaneous current measurements and tracer experiments, and it is possible to compare the results.

Besides that, one can introduce the Hay-Pasquill hypothesis [1959], which states principal similarity in the shape of the Lagrangian and the Eulerian correlation function and introduce the transformation $R_{\mathrm{L}}(\gamma t)=R_{\mathrm{E}}(t)$. Hay and Pasquill obtained $\gamma=4$ in the atmosphere under a wide range of stability conditions, but the spread is considerable. Schott and Quadfasel [1979] obtained $\gamma=1.4 \pm 0.4$ for rhodamin experiments in the Baltic.

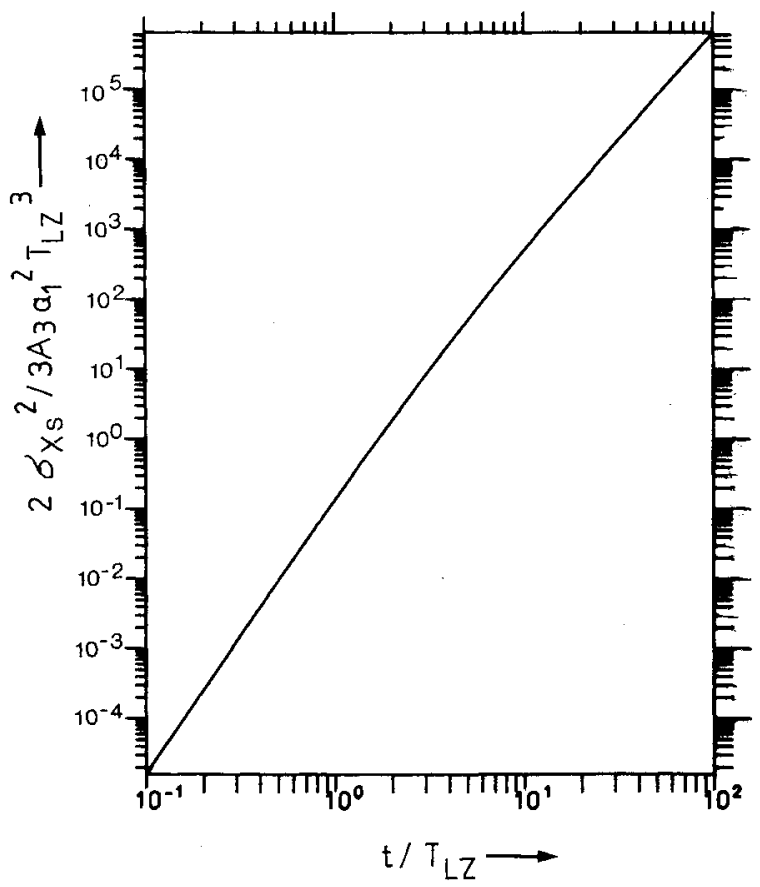

Fig. 3. Variance generated by shear dispersion $\sigma_{X S}^{2} /\left(2 / 3 A_{3} a_{1}^{2} T_{\mathrm{LZ}}^{3}\right)$ versus time $t / T_{\mathrm{L} Z}$ (for definitions see section 3.4) 


\subsection{Spatial extended source}

It is impossible to materialize a momentaneous point source exactly. How to calculate the moments in the case of a spatially extended source is shown by a simple example. Assuming a current varying linearly with depth, the origin of the coordinate system is chosen in such a way that the first moments of the distribution vanish. The solution of $\left(5^{\mathrm{I}}\right)$ is:

$$
\begin{aligned}
\sigma_{Z}^{2}= & \left\{z^{2}\right\} \\
= & \left\{z^{2}(t=0)\right\}+2 \int_{0}^{t} A_{Z 0} \mathrm{~d} t^{\prime} \\
\{x z\}= & \{x z(t=0)\}+\int_{0}^{t} a_{1}\left\{z^{2}(t=0)\right\} \mathrm{d} t^{\prime}+2 a_{1} \int_{0}^{t} \mathrm{~d} t^{\prime} \int_{0}^{t^{\prime}} \mathrm{d} t^{\prime \prime} A_{Z 0} \\
\sigma_{X}^{2}= & \left\{x^{2}\right\} \\
= & \left\{x^{2}(t=0)\right\}+2 a_{1} \int_{0}^{t} a_{1}\{x Z(t=0)\} \mathrm{d} t^{\prime} \\
& +2 \int_{0}^{t} \mathrm{~d} t^{\prime} a_{1} \int_{0}^{t^{\prime}} \mathrm{d} t^{\prime \prime} a_{1}\left\{z^{2}(t=0)\right\} \\
& +4 \int_{0}^{t} \mathrm{~d} t^{\prime} a_{1} \int_{0}^{t^{\prime}} \mathrm{d} t^{\prime \prime} a_{1} \int_{0}^{t^{\prime \prime}} \mathrm{d} t^{\prime \prime \prime} A_{Z 0} \\
& +2 \int_{0}^{t} \mathrm{~d} t^{\prime} A_{X 0} .
\end{aligned}
$$

Assuming the current to be steady and $\left\{x^{2}(t=0)\right\}=\{x z(t=0)\}=0,(20)$ yields the term $a_{1}^{2}\left\{z^{2}(t=0)\right\} t^{2}$ in addition to the shear generated part of the variance. This result was obtained by Kullenberg [1972] from geometrical arguments.

\subsection{Expansion to three dimensional space}

There are many ways to expand the aforementioned calculations to three dimensional space. Some of them are shown in brief.

a) The current as a function of $z$ and $t$ has only one component, which will be taken as being the $x$ direction. The moments containing merely $x$ and $z$ remain the same as in the two dimensional case. The variance in $y$ direction is given by $\sigma_{Y}^{2}=2 A_{Y 0} t$.

b) The current has only one component in the $x$ direction but is a function of $y, z$, and $t$ :

$$
u=\sum_{v=0}^{N}\left(a_{v} z^{v}+b_{v} y^{v}\right)
$$

The resulting reduction formula is:

$$
\begin{aligned}
\frac{\mathrm{d}}{\mathrm{d} t}\left\{x^{k} y^{l} z^{m}\right\}= & k \sum_{v=0}^{N}\left(a_{v}\left\{x^{k-1} y^{l} z^{m+v}\right\}+b_{v}\left\{x^{k-1} y^{l+v} z^{m}\right\}\right) \\
& +k(k-1) A_{X O}\left\{x^{k-2} y^{l} z^{m}\right\} \\
& +l(l-1) A_{Y O}\left\{x^{k} y^{l-2} z^{m}\right\} \\
& +m(m-1) A_{Z 0}\left\{x^{k} y^{l} z^{m-2}\right\}
\end{aligned}
$$


Assuming a steady current and the initial conditions (6), one obtains:

$$
\begin{aligned}
\sigma_{X}^{2}= & 2 A_{X 0} t \\
& +\frac{2}{3} a_{1}^{2} A_{Z 0} t^{3}+\left(\frac{4}{3} a_{2}^{2}+5 a_{1} a_{3}\right) A_{Z 0}^{2} t^{4}+\frac{72}{5} a_{3}^{2} A_{Z 0}^{3} t^{5} \\
& +\frac{2}{3} b_{1}^{2} A_{Y 0} t^{3}+\left(\frac{4}{3} b_{2}^{2}+5 b_{1} b_{3}\right) A_{Y 0}^{2} t^{4}+\frac{72}{5} b_{3}^{2} A_{Y 0}^{3} t^{5} .
\end{aligned}
$$

For the linear case Carter and Okubo [1965] obtained:

$$
\sigma_{X}^{2}=2 A_{X 0} t+\frac{1}{6} a_{1}^{2} A_{Z 0} t^{3}+\frac{2}{3} b_{1}^{2} A_{Y O} t^{3}
$$

(in my notation), which is in contradiction to the aforementioned result (21). This is explained by an error of Carter and Okubo in computing the variance (see Appendix 3).

c) The current is dependent upon depth and time only:

$$
u=\sum_{\nu=0}^{N} a_{\nu} z^{\nu} \text { and } v=\sum_{\nu=0}^{N} b_{\nu} z^{\nu} .
$$

The resulting reduction formula is:

$$
\begin{aligned}
\frac{\mathrm{d}}{\mathrm{d} t}\left\{x^{k} y^{l} z^{m}\right\}= & k \sum_{v=0}^{N} a_{v}\left\{x^{k-1} y^{l} z^{m+v}\right\} \\
& +l \sum_{v=0}^{N} b_{v}\left\{x^{k} y^{l-1} z^{m+v}\right\} \\
& +k(k-1) A_{X 0}\left\{x^{k-2} y^{l} z^{m}\right\} \\
& +l(l-1) A_{Y 0}\left\{x^{k} y^{l-2} z^{m}\right\} \\
& +m(m-1) A_{Z 0}\left\{x^{k} y^{l} z^{m-2}\right\}
\end{aligned}
$$

With a steady current, and the initial conditions (6), this yields:

$$
\begin{aligned}
\sigma_{X}^{2} & =2 A_{X 0} t+\frac{2}{3} a_{1}^{2} A_{Z 0} t^{3}+\left(\frac{4}{3} a_{2}^{2}+5 a_{1} a_{3}\right) A_{Z 0}^{2} t^{4}+\frac{72}{5} a_{3}^{2} A_{Z 0}^{3} t^{5} \\
\sigma_{Y}^{2} & =2 A_{Y 0} t+\frac{2}{3} b_{1}^{2} A_{Z 0} t^{3}+\left(\frac{4}{3} b_{2}^{2}+5 b_{1} b_{3}\right) A_{Z 0}^{2} t^{4}+\frac{72}{5} b_{3}^{2} A_{Z 0}^{3} t^{5} \\
\sigma_{Z}^{2} & =2 A_{Z 0} t .
\end{aligned}
$$

Kullenberg [1972 and 1974] gives the following results for $u=\left(a_{10}+a_{1} \cos (\omega t) \cdot z\right.$ and $v=b_{1} \sin (\omega t) \cdot z$ with (6) as initial conditions:

$$
\begin{aligned}
\sigma_{X \mathrm{~K}}^{2}= & \frac{2}{3} A_{Z 0} a_{10}^{2} t^{3}+\frac{a_{1}^{2}}{\omega^{2}} A_{Z 0} t-\frac{a_{1}^{2}}{2 \omega^{3}} A_{Z 0} \sin 2 \omega t \\
& -4 \frac{a_{10} a_{1}}{\omega^{2}} A_{Z 0} t \cos 2 \omega t+4 \frac{a_{10} a_{1}}{\omega^{3}} A_{Z 0} \sin \omega t \\
\sigma_{Y \mathrm{~K}}^{2}= & \frac{9}{8} \frac{b_{1}^{2}}{\omega^{2}} A_{Z 0} t+\frac{b_{1}^{2} a_{10}}{a_{1}^{2} \omega^{2}}\left(2 a_{10}-a_{1}\right) A_{Z 0} t \\
& +A_{Z 0} \frac{b_{1}^{2}}{2 \omega^{3}} \sin 2 \omega t-A_{Z 0} \frac{b_{1}^{2}\left(a_{1}-4 a_{10}\right)}{a_{1} \omega^{3}} \sin \omega t
\end{aligned}
$$


$a_{1}=0$ is excluded. The present method yields a contradictory result:

$$
\begin{aligned}
\sigma_{X}^{2}= & \frac{2}{3} A_{Z 0} a_{10}^{2} t^{3}+2 A_{Z 0} a_{10} a_{1} \cdot\left(\frac{t^{2}}{\omega} \sin \omega t-\frac{t}{\omega^{2}}(1-\cos \omega t)\right) \\
& +2 A_{Z 0} \frac{a_{1}^{2}}{\omega^{2}}\left(t\left(1-\frac{1}{2} \cos 2 \omega t\right)+\frac{3}{4} \frac{\sin 2 \omega t}{\omega}-2 \frac{\sin \omega t}{\omega}\right) \\
\sigma_{Y}^{2}= & 2 A_{Z 0} \frac{b_{1}^{2}}{\omega^{2}}\left(t+\frac{t}{2} \cos 2 \omega t-\frac{3}{4} \frac{\sin 2 \omega t}{\omega}\right) .
\end{aligned}
$$

Kullenberg's result must be wrong; because for the limit $a_{1} \rightarrow 0$ and both $b_{1}$ and $a_{10} \neq 0, \sigma_{Y K}^{2}$ increases to infinity.

\section{Depth dependence of the vertical exchange coefficient}

This case will again be treated in two dimensions. The three dimensional case can easily be derived analogous to the cases in the foregoing section. (6) is always taken as initial condition and steady currents are assumed.

4.1 Vertical exchange coefficient varying linearly with depth

In this case, (5) reduces to

$$
\begin{aligned}
\frac{\mathrm{d}}{\mathrm{d} t}\left\{x^{k} z^{m}\right\}= & k \sum_{\nu=1}^{N} a_{v}\left\{x^{k-1} z^{m+\nu}\right\} \\
& +k(k-1) A_{X 0}\left\{x^{k-2} z^{m}\right\} \\
& +m(m-1) A_{Z 0}\left\{x^{k} z^{m-2}\right\} \\
& +m^{2} A_{Z 1}\left\{x^{k} z^{m-1}\right\} .
\end{aligned}
$$

This set of ordinary differential equations can be solved by successive time integration. The result obtained for the variances is:

$$
\begin{aligned}
\sigma_{Z}^{2}= & 2 A_{Z 0} t+A_{Z 1}^{2} t^{2} \\
\sigma_{X}^{2}= & 2 A_{X 0} t+a_{1}^{2}\left(\frac{2}{3} A_{Z 0} t^{3}+\frac{1}{6} A_{Z 1}^{2} t^{4}\right) \\
& +a_{1} a_{2}\left(4 A_{Z 0} A_{Z 1} t^{4}+\frac{14}{15} A_{Z 1}^{3} t^{5}\right) \\
& +a_{1} a_{3}\left(5 A_{Z 0}^{2} t^{4}+\frac{82}{5} A_{Z 0} A_{Z 1}^{2} t^{5}+\frac{16}{5} A_{Z 1}^{4} t^{6}\right) \\
& +a_{2}^{2}\left(\frac{4}{3} A_{Z 0}^{2} t^{4}+\frac{116}{15} A_{Z 0} A_{Z 1}^{2} t^{5}+\frac{8}{5} A_{Z 1}^{4} t^{6}\right) \\
& +a_{2} a_{3}\left(\frac{186}{5} A_{Z 0}^{2} A_{Z 1} t^{5}+\frac{376}{5} A_{Z 0} A_{Z 1}^{3} t^{6}+\frac{456}{35} A_{Z 1}^{5} t^{7}\right) \\
& +a_{3}^{2}\left(\frac{72}{5} A_{Z 0}^{3} t^{5}+\frac{831}{5} A_{Z 0}^{2} A_{Z 1}^{2} t^{6}+\frac{1044}{5} A_{Z 0} A_{Z 1}^{4} t^{7}+\frac{2169}{70} A_{Z 1}^{6} t^{8}\right) .
\end{aligned}
$$

The results for the moments necessary to calculate the variances are given in Appendix 4 . 
4.2. Vertical exchange coefficient varying as $z^{2}$

With $A_{Z 1}=0$ (the case $A_{Z 1} \neq 0$ shall not be discussed here because of the rather tedious and voluminous calculations, the result is given in Mikolajewicz [1984]), (5) reduces to:

$$
\begin{aligned}
\frac{\mathrm{d}}{\mathrm{d} t}\left\{x^{k} z^{m}\right\}= & k \sum_{\nu=0}^{N} a_{v}\left\{x^{k-1} z^{m+v}\right\} \\
& +k(k-1) \sum_{v=0}^{N_{X}} A_{X v}\left\{x^{k-2} z^{m+v}\right\} \\
& +m(m-1) A_{Z 0}\left\{x^{k} z^{m-2}\right\} \\
& +m(m+1) A_{Z 2}\left\{x^{k} z^{m}\right\}
\end{aligned}
$$

This describes a closed system of ordinary differential equations, which can be solved successively by the method of variation of the constant. For the variances one obtains:

$$
\begin{aligned}
\sigma_{Z}^{2}= & \frac{1}{3} \frac{A_{Z 0}}{A_{Z 2}}\left(\mathrm{e}^{6 A_{Z 2} t}-1\right) \\
\sigma_{X}^{2}= & 2 A_{X 0} t \\
& +A_{X 2} \frac{A_{Z 0}}{A_{Z 2}^{2}}\left(\frac{1}{9} \mathrm{e}^{6 A_{Z 2} t}-\frac{1}{9}+\frac{2}{3} A_{Z 2} t\right) \\
+ & a_{1}^{2} \frac{A_{Z 0}}{A_{Z 2}^{3}}\left[\frac{1}{36}\left(\mathrm{e}^{6 A_{Z 2} t}-1\right)-\frac{1}{4}\left(\mathrm{e}^{2 A_{Z 2} t}-1\right)+\frac{1}{3} A_{Z 2} t\right] \\
+ & a_{1} a_{3} \frac{A_{Z 0}^{2}}{A_{Z 2}^{4}}\left[\frac{13}{8400}\left(\mathrm{e}^{20 A_{Z 2} t}-1\right)-\frac{1}{240}\left(\mathrm{e}^{12 A_{Z 2} t}-1\right)-\frac{1}{28}\left(\mathrm{e}^{6 A_{Z 2} t}-1\right)\right. \\
& \left.+\frac{19}{60}\left(\mathrm{e}^{2 A_{Z 2} t}-1\right)-\frac{2}{5} A_{Z 2} t\right] \\
+ & a_{2}^{2} \frac{A_{Z 0}^{2}}{A_{Z 2}^{4}}\left[\frac{3}{4900}\left(e^{20 A_{Z 2} t}-1\right)-\frac{1}{324}\left(\mathrm{e}^{12 A_{Z 2} t}-1\right)\right. \\
& \left.+\frac{50}{3969}\left(\mathrm{e}^{6 A_{Z 2} t}-1\right)-\frac{4}{189} A_{Z 2} t \mathrm{e}^{A_{Z 2} t}-\frac{4}{135} A_{Z 2} t\right] \\
+ & a_{3}^{2} \frac{A_{Z 0}^{3}}{A_{Z 2}^{5}}\left[\frac{1}{29106}\left(e^{42 A_{Z 2} t}-1\right)-\frac{17}{15400}\left(\mathrm{e}^{20 A_{Z 2} t}-1\right)\right. \\
& +\frac{1}{360}\left(\mathrm{e}^{12 A_{Z 2} t}-1\right)+\frac{2}{189}\left(\mathrm{e}^{6 A_{Z 2} t}-1\right) \\
& \left.-\frac{1}{10}\left(\mathrm{e}^{2 A_{Z 2} t}-1\right)+\frac{13}{105} A_{Z 2} t\right] .
\end{aligned}
$$

The moments necessary for the calculation of the variances are given in Appendix 5. In the limit $A_{Z 2} \rightarrow 0$, this yields (9). The time $t$ only appears as the product $A_{Z 2} t . A_{Z 2}^{-1}$ can be interpreted as a timescale characteristic for vertical mixing. If $\left|A_{Z 2} t\right| \ll 1$, the tracer does not "feel" the boundaries, this case is equivalent to the case with a constant vertical exchange coefficient. 
In the following, it is assumed that $A_{Z 2}=-A_{Z 0} / h^{2}$. Thus, the vertical exchange coefficient is given by:

$$
A_{33}(z)=A_{Z 0}\left(1-\frac{z^{2}}{h^{2}}\right)
$$

$A_{33}$ becomes 0 for $|z|=h$ and negative for $|z|>h$. This is equivalent to solid boundaries at $|z|=h$ and thus forming a channel. To prove this, the vertical moments $\left\{z^{2 n}\right\}$ for large diffusion times $\left|A_{Z 2} t\right| \geqslant 1$ have been calculated. The moments converge with the limits:

$$
\begin{aligned}
\lim _{Z_{Z 2} t \rightarrow-\infty}\left\{z^{2}\right\} & =\frac{1}{3} h^{2} \\
\lim _{\boldsymbol{A}_{Z 2} t \rightarrow-\infty}\left\{z^{4}\right\} & =\frac{1}{5} h^{4} \\
\lim _{\boldsymbol{A}_{Z 2} t \rightarrow-\infty}\left\{z^{6}\right\} & =\frac{1}{7} h^{6},
\end{aligned}
$$

which are equal to the moments that a distribution with constant concentration between $-h$ and $+h$ and 0 outwards would yield.

In the case of a channel with a tracer equally distributed over depth, it is known (e.g. Taylor [1953] and Bowden [1965]) that the variance increases linearly with time, resulting in a constant effective diffusivity

$$
A_{X \text { eff }}=\frac{1}{2} \frac{\mathrm{d} \sigma_{X}^{2}}{\mathrm{~d} t}
$$

(25) yields an effective diffusivity in the large time limit $A_{Z 2} t \ll-1$ of:

$$
A_{X \text { eff }}=A_{X 0}-\frac{1}{3} A_{X 2} h^{2}+\frac{1}{6} \frac{a_{1}^{2} h^{4}}{A_{Z 0}}+\left(\frac{1}{5} a_{1} a_{3}+\frac{2}{135} a_{2}^{2}\right) \frac{h^{6}}{A_{Z 0}}+\frac{13}{210} a_{3}^{2} \frac{h^{8}}{A_{Z 0}}
$$

which is in agreement with the result one obtains using Bowden's method for the same depth dependence of current and vertical exchange coefficient.

To investigate the influence of the nonlinearities of the current profile and the impenetrable boundaries on the variance, a "Gedanken"experiment was conducted. The current is assumed to be steady and the stratification to be neutral. By assuming that the vertical exchange coefficients for matter and momentum have the same shape, Elder [1959] has shown that a depth dependence equal to $A_{33}=K \cdot\left(1-z^{2} / h^{2}\right)$ leads to a logarithmic current profile, if the stress varies linearly with depth:

$$
\frac{u(z)}{u_{*}}=\ln \frac{z+h}{z_{0}} \quad u_{*} \ln \frac{2 \mathrm{~h}}{z_{0}}=u_{0} .
$$

The arbitrary value of $1 / 40$ of the depth of the channel $H=2 h$ is now chosen for the friction depth $z_{0} \cdot u_{0}$ is the maximum velocity at the upper layer. To fit the present models, $u(z)$ was expanded as a Taylor series at $z=0$ :

$$
\frac{u(z)}{u_{0}}=\frac{\ln H-\ln 2 z_{0}+\sum_{v=1}^{N} \frac{1}{v}(-1)^{v-1}\left(\frac{2(z+h)}{H}\right)^{v}}{\ln H-\ln z_{0}}
$$


The result for various $N$ is shown in Fig. 4. Fig. 5 illustrates the non-dimensional relationship between the shear generated part of the horizontal variance $\sigma_{X s}^{2}$ and the time $t$. The following 4 cases are given:

$$
\begin{array}{ll}
u=\sum_{v=0}^{3} a_{v} z^{v} & A_{33}=K\left(1-\frac{z^{2}}{h^{2}}\right) \\
u=\sum_{v=0}^{1} a_{v} z^{v} & A_{33}=K\left(1-\frac{z^{2}}{h^{2}}\right) \\
u=\sum_{v=0}^{1} a_{v} z^{v} & A_{33}=K \\
u=\sum_{v=0}^{3} a_{v} z^{v} & A_{33}=K .
\end{array}
$$

Case 3 is equal to Okubo's model [1967]. For small diffusion times $K t / h^{2}<0.1$, Okubo's model provides quite a good approximation of the variance $\sigma_{X s}^{2}$, resulting in an increase of $\sigma_{X s}^{2}$ as $t^{3}$. For large times, the non-linearity of the current profile generates a greater increase of the variance (case 4), whereas the effect of the boundaries results in a diminished increase (case 2). Those two effects compensate each other, so that for times less than $K t / h^{2}<0.3$, case 1 and 3 are nearly equal. For larger diffusion times, the variance in the cases 1 and 2 increases linearly with time, as has been described by Taylor [1953] and Bowden [1965].

In Fig. 6, the non-dimensional effective exchange coefficient is shown as a function of non-dimensional time. For diffusion times $t>3 h^{2} / K, A_{X \text { eff }}$ is constant.

For a parabolic vertical exchange coefficient and for a current profile of the shape

$$
u=\sum_{v=0}^{N} a_{v} z^{v}
$$

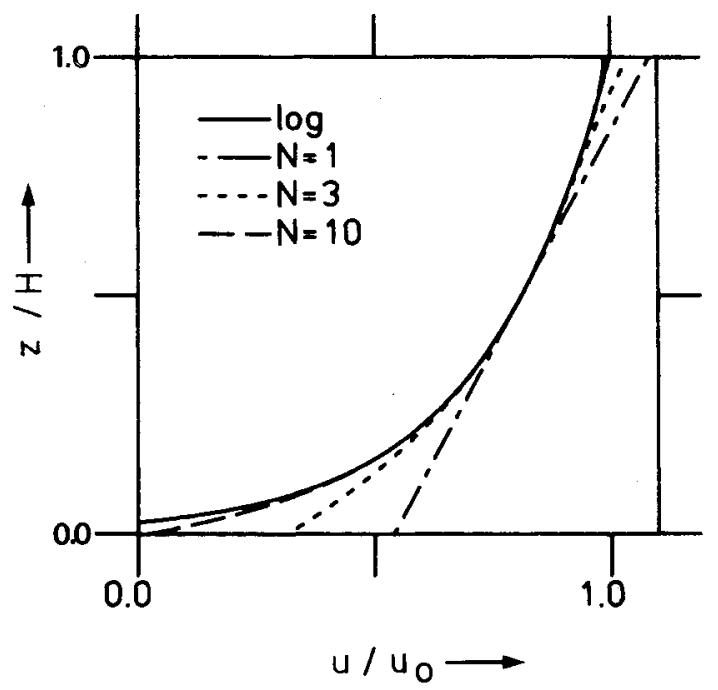

Fig. 4. Velocity $u$ versus various approximations versus depth $z / H$ (for definitions see section 4.2) 


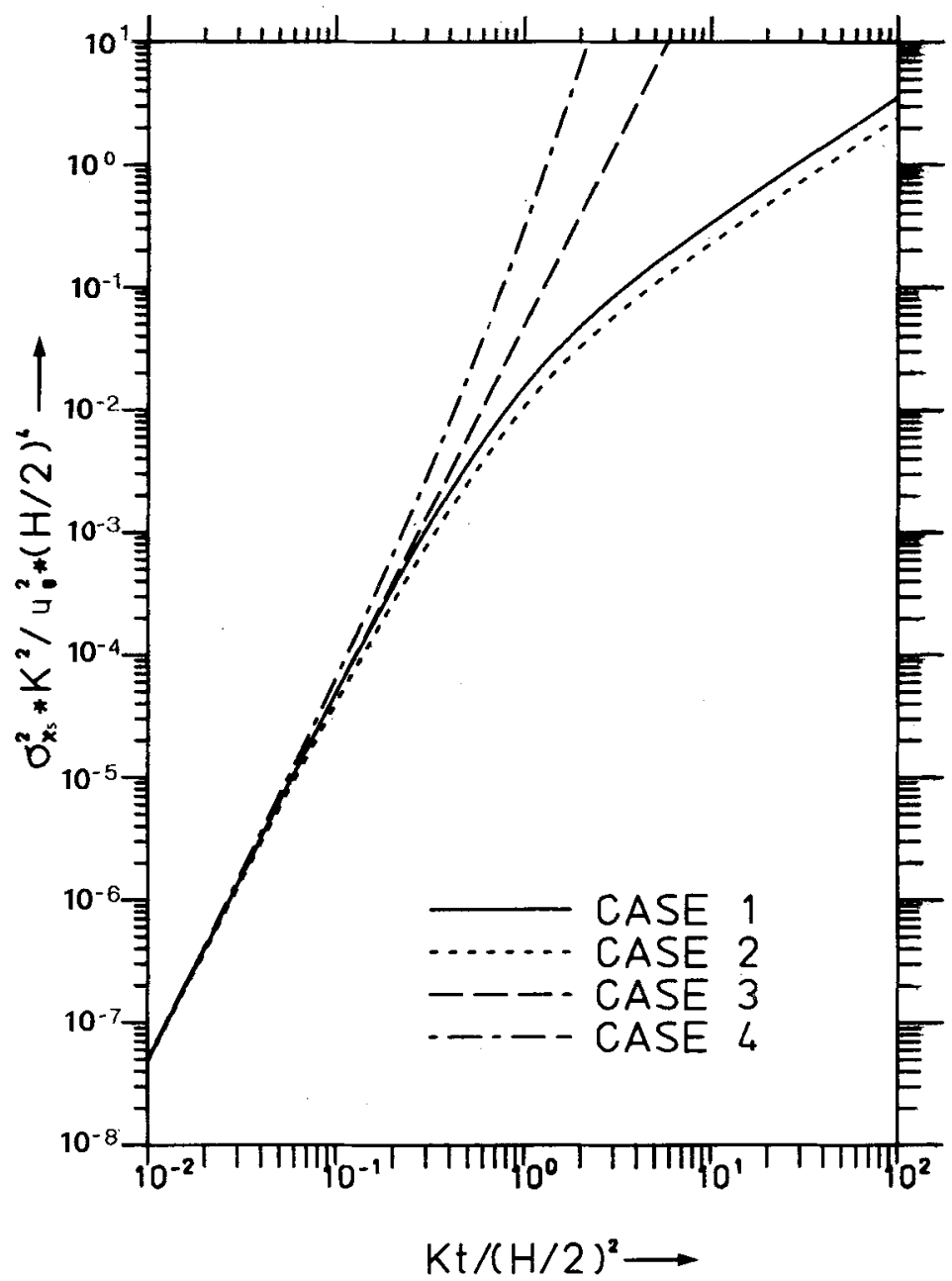

Fig. 5. Shear generated part of the variance $\sigma_{X \mathrm{~s}}^{2} K^{2} /\left(u_{0}^{2} h^{4}\right)$ versus time $K t / h^{2}$ (for definitions see section 4.2) 


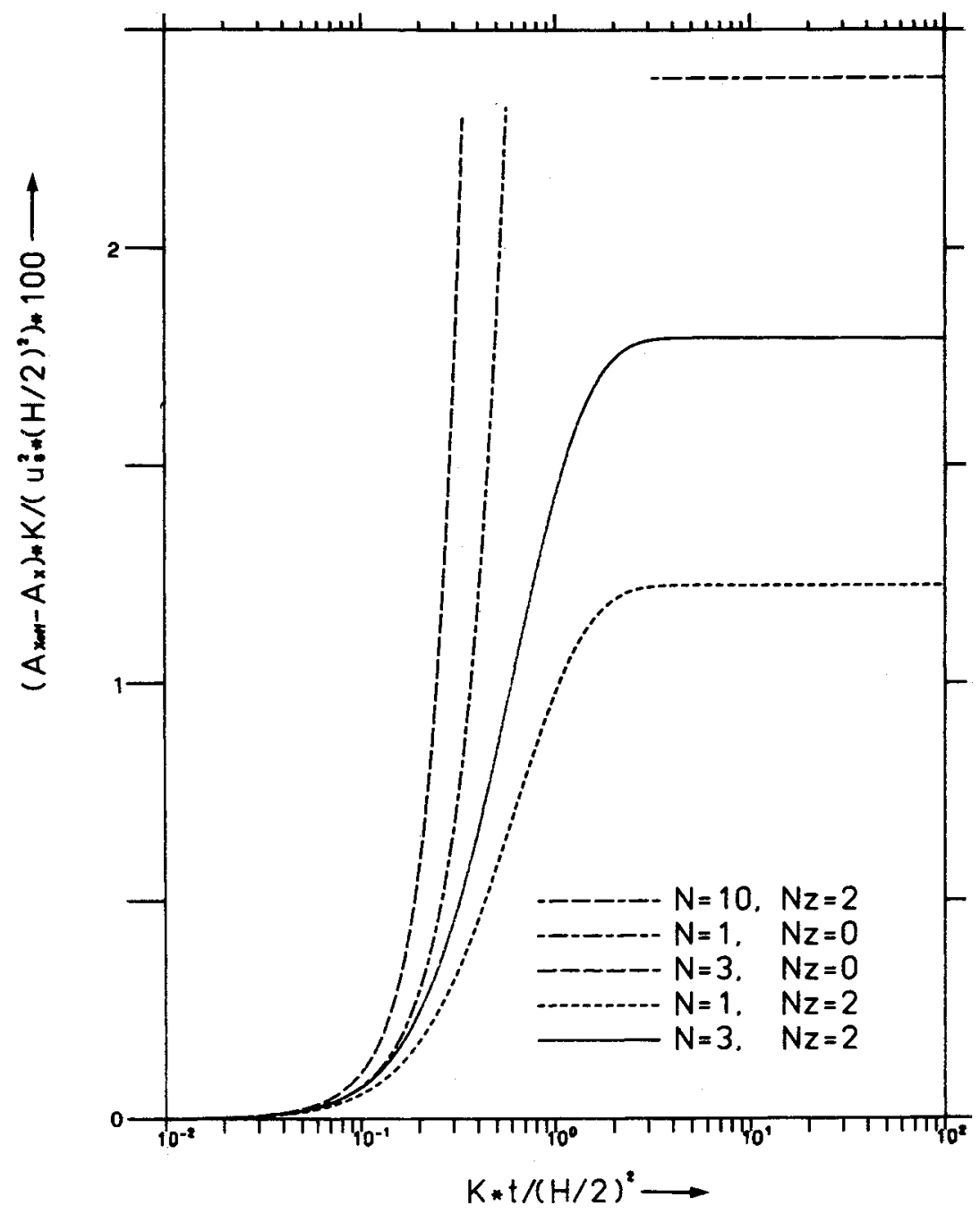

Fig. 6. Effective exchange coefficient $\left(A_{X \text { eff }}-A_{X}\right) K /\left(u_{0}^{2} h^{2}\right)$ (for definitions see section 4.2) 
Bowden's model [1965] yields:

$$
\begin{aligned}
A_{X \text { eff }}= & \frac{h^{2}}{K}\left[\left(\sum_{\eta=1}^{N} \frac{b_{\eta}}{\eta+1}\right) \cdot \sum_{\mu=1}^{N}\left(\sum_{\nu=\mu}^{N} \frac{b_{v}}{v+1}\right) \frac{1}{\mu(\mu+1)}\right. \\
& -\sum_{\eta=1}^{N} \sum_{\mu=1}^{N}\left(\sum_{\nu=\mu}^{N} \frac{b_{\nu}}{v+1}\right) \frac{b_{\eta}}{\mu(\eta+\mu+1)}
\end{aligned}
$$

where the $b_{\mu}$ are given by:

$$
b_{\mu}=\sum_{\nu=\mu}^{N} a_{\nu}(-1)^{\nu-\mu}\left(\begin{array}{l}
v \\
\mu
\end{array}\right) 2^{\mu} .
$$

The results are given below for various $N$ :

\begin{tabular}{rc}
\hline$N$ & $A_{X \mathrm{eff}} \cdot K /\left(u_{0}^{2} \cdot h^{2}\right)$ \\
\hline 1 & 0.0124 \\
3 & 0.0179 \\
10 & 0.0239 \\
\hline
\end{tabular}

The relative error of $A_{X \text { eff }}$ generated by the approximation of the current is about $25 \%$ with $N=3$, whereas it is about $50 \%$ with a linear current profile.

\section{Hypothetical dependence of the variance upon time}

In this section, the possible dependence of variance upon time-according to the results presented - is reviewed in brief.

The horizontal spread of tracers is due to two additive effects: shear dispersion and horizontal turbulent diffusion. The horizontal turbulent diffusion produces an increase of $\sigma_{X \mathrm{~h}}^{2}$ as $t^{2}$ for small diffusion times $\left(t \ll 2 T_{\mathrm{LX}}\right)$ and a linear increase in time for $t \gg 2 T_{\mathrm{L} X}$. The contribution of shear dispersion $\sigma_{X \mathrm{~s}}^{2}$ increases as $t^{4}$ for as long as $t \ll 4 T_{\mathrm{LZ}}$. For intermediate diffusion times $\left(4 T_{\mathrm{L} Z} \ll t \ll h^{2} / A_{Z 0}\right)$, it increases as $t^{3}$, and for large times $\left(t \gg h^{2} / A_{Z 0}\right)$ it increases linearly with time.

Summarized this might give the following picture: Firstly $\sigma_{X}^{2}$ increases as $t^{2}$, because of horizontal turbulent diffusion. If the condition

$$
t_{\mathrm{c}}=2.5 \sqrt{\frac{\overline{v^{\prime 2}}}{\overline{w^{\prime 2}}}} \ll 4 T_{\mathrm{L} Z}
$$

is fulfilled, the variance first grows as $t^{4}$ for $t_{\mathrm{c}} \ll t \ll 4 T_{\mathrm{L} Z}$ and then as $t^{3}$ for $4 T_{\mathrm{LZ}} \ll t \ll h^{2} / A_{Z 0}$. If $t_{\mathrm{c}} \ll 4 T_{\mathrm{L} Z}$, then the $t^{4}$ range is non-existent. In the literature, the $t^{4}$ range is not mentioned. This can be explained by the fact that either the conditions for its existence were not fulfilled in some experiments, or that it has not been recognized, because it is unusual to distinguish between several ranges, and very often Okubo's model [1967] has been used, and the residuum has been explained by the uncertainties of the measurements. Okubo [1971] considers mass balances as low as $50 \%$ to be permissible. For very large diffusion times $\left(t \gg h^{2} / A_{Z 0}\right)$ the variance increases linearly in time.

In Fig. 7, the consequences of the aforementioned considerations are shown. For $\overline{u^{\prime 2}}$, $\overline{w^{\prime 2}}, T_{\mathrm{L} Z}$ and $|d v / \mathrm{d} z|$, values obtained from a rhodamin experiment in the open Baltic described in Mikolajewicz [1984] - are used. $T_{\mathrm{LX}}$ has been chosen to be about 5 days. 


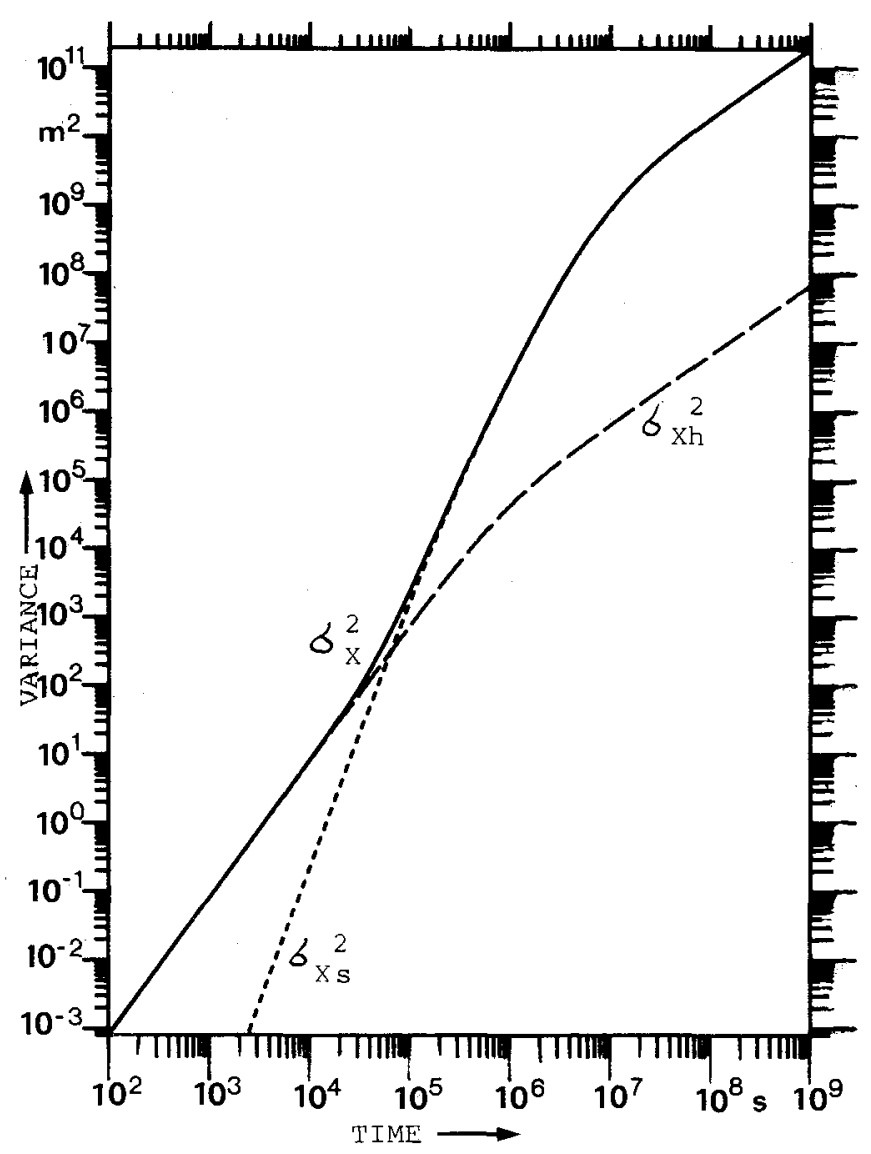

Fig. 7. Hypothetical dependence of the variance on time.

Coefficients are taken from a rhodamin experiment in the Baltic described in Mikolajewicz [1984]

\section{Appendix 1}

A relative simple method of calculating an approximative distribution if only a limited number of moments are known is described here.

Due to the fact that $\exp \left(-x^{2} / \sigma_{X}^{2}-y^{2} / \sigma_{Y}^{2}-z^{2} / \sigma_{Z}^{2}\right)$ is different from 0 for all $x, y$ and $z-$ if only the variances are different from 0 - the distribution can be expressed in the following way:

$$
C(x, y, z)=f(x, y, z) \exp \frac{1}{2}\left(-x^{2} / \sigma_{X}^{2}-y^{2} / \sigma_{Y}^{2}-z^{2} / \sigma_{Z}^{2}\right) .
$$

where $f$ is a real function. If $C$ has derivatives of all orders, then $f$ has too, and can be expressed as a power series expansion (Bronstein and Semandjajew [1979]):

$$
f(x, y, z)=\sum_{p}^{\infty} \sum_{q}^{\infty} \sum_{r}^{\infty} g_{p q r} x^{p} y^{q} z^{r}
$$

The moments are defined as:

$$
\left\{x^{k} y^{l} z^{m}\right\}=\iint_{-\infty}^{\infty} \int_{\infty} \mathrm{d} V C x^{k} y^{l} z^{m}
$$


With (A2) this yields:

$$
\begin{aligned}
\left\{x^{k} y^{l} z^{m}\right\} & =\iint_{-\infty}^{\infty} \int_{-\infty} \mathrm{d} V x^{k} y^{l} z^{m}\left(\sum_{p}^{\infty} \cdot \sum_{q}^{\infty} \sum_{r}^{\infty} g_{p q r} \cdot \exp \frac{1}{2}\left(-\frac{x^{2}}{\sigma_{X}^{2}}-\frac{y^{2}}{\sigma_{Y}^{2}}-\frac{z^{2}}{\sigma_{Z}^{2}}\right) x^{p} y^{q} z^{r}\right) \\
& =\sum_{p}^{\infty} \sum_{q}^{\infty} \sum_{r}^{\infty} g_{p q r} \iint_{-\infty}^{\infty} \int_{\infty} \mathrm{d} V x^{k+p} y^{l+q} z^{m+r} \exp \frac{1}{2}\left(-\frac{x^{2}}{\sigma_{X}^{2}}-\frac{y^{2}}{\sigma_{Y}^{2}}-\frac{z^{2}}{\sigma_{Z}^{2}}\right) .
\end{aligned}
$$

The integrals are clearly defined and yield (Gröbner and Hofreiter [1958]):

$$
\begin{aligned}
\left\{x^{k} y^{l} z^{m}\right\}=\sum_{p} \sum_{q}^{\infty} \sum_{r}^{\infty} g_{p q r} & \frac{\left(1+(-1)^{k+r}\right)}{2} \frac{1 \cdot 3 \cdot 5 \ldots(k+p-1)}{\pi} \sqrt{\left.2 \sigma_{X}^{2}\right)^{\frac{k+p+p}{2}}} \\
& \cdot \frac{\left(1+(-1)^{l+q}\right)}{2} \frac{1 \cdot 3 \cdot 5 \ldots(l+q-1)}{2^{\frac{l+q}{2}}} \sqrt{\pi}\left(2 \sigma_{Y}^{2}\right)^{\frac{l+q+1}{2}} \\
& \cdot \frac{\left(1+(-1)^{m+r}\right)}{2} \frac{1 \cdot 3 \cdot 5 \ldots(m+r-1)}{2^{\frac{m+r}{2}}} \sqrt{\pi}\left(2 \sigma_{Z}^{2}\right)^{\frac{m+r+1}{2}} .
\end{aligned}
$$

Stopping the summation at $N \geqq p+q+r$ results in:

$$
\begin{aligned}
\left\{x^{k} y^{l} z^{m}\right\} \approx \sum_{p=0}^{N} \sum_{q=0}^{N-p} \sum_{r=0}^{N-p-q} & g_{p q r} \cdot \pi^{\frac{3}{2}} \cdot \sigma_{X}^{k+p+1} \cdot \sigma_{Y}^{l+q+1} \cdot \sigma_{Z}^{m+r+1} \cdot 2^{-1.5} \\
& \cdot\left(1+(-1)^{k+p}\right) \cdot\left(1+(-1)^{l+q}\right) \cdot\left(1+(-1)^{m+r}\right) \\
& \cdot 1 \cdot 3 \cdot 5 \cdot \ldots(k+p-1) \cdot 1 \cdot 3 \cdot 5 \cdot \ldots \cdot(l+q-1) \\
& \cdot 1 \cdot 3 \cdot 5 \cdot \ldots \cdot(m+r-1) .
\end{aligned}
$$

If the moments are known for all $k, l$ and $m$, with $k+l+m \leqq N$, a closed system of linear equations is defined that can be solved by the methods of linear algebra.

$C_{N}$ is defined as:

$$
C_{N}:=\sum_{p=0}^{N} \sum_{q=0}^{N-p} \sum_{r=0}^{N-p-q} g_{p q r}(N) \cdot x^{p} y^{q} z^{r} \cdot \exp \frac{1}{2}\left(-\frac{x^{2}}{\sigma_{X}^{2}}-\frac{y^{2}}{\sigma_{Y}^{2}}-\frac{z^{2}}{\sigma_{Z}^{2}}\right)
$$

and $\lim _{N \rightarrow \infty} C_{N}=C$.

The convergence can be improved by a transformation of the co-ordinate system, thus eliminating the first moments and the mixed second moments.

\section{Appendix 2}

The complete solution for the oscillating current is:

$$
\begin{aligned}
\left\{z^{2 n+1}\right\}= & 0 \\
\left\{z^{2 n}\right\}= & \frac{(2 n) !}{n !} A_{Z 0}^{n} t^{n} \\
\{x\}= & -\frac{a_{0}}{\omega}(\cos (\omega t+\beta)-\cos \beta) \\
& +2 A_{Z 0} \frac{a_{2}}{\omega^{2}}(\sin (\omega t+\beta)-\sin \beta)-2 A_{Z 0} t \frac{a_{2}}{\omega} \cos (\omega t+\beta)
\end{aligned}
$$




$$
\begin{aligned}
& \{x z\}=2 A_{z 0} \frac{a_{1}}{\omega^{2}}(\sin (\omega t+\beta)-\sin \beta)-2 A_{z 0} t \frac{a_{1}}{\omega} \cos (\omega t+\beta) \\
& +12 A_{Z 0}^{2} \frac{a_{3}}{\omega}\left(2 \frac{t}{\omega} \sin (\omega t+\beta)-t^{2} \cos (\omega t+\beta)+\frac{2}{\omega^{2}}(\cos (\omega t+\beta)-\cos \beta)\right) \\
& \left\{x z^{2}\right\}=-2 A_{Z 0} t \frac{a_{0}}{\omega}(\cos (\omega t+\beta)-\cos \beta)-12 A_{Z 0}^{2} t^{2} \frac{a_{2}}{\omega} \cos (\omega t+\beta) \\
& -4 A_{Z 0}^{2} t \frac{a_{2}}{\omega^{2}} \sin \beta+20 A_{Z 0}^{2} t \frac{\dot{a}_{2}}{\omega^{2}} \sin (\omega t+\beta) \\
& +16 A_{Z 0}^{2} \frac{a_{2}}{\omega^{3}}(\cos (\omega t+\beta)-\cos \beta) \\
& \left\{x z^{3}\right\}=-12 A_{Z 0}^{2} t^{2} \frac{a_{1}}{\omega} \cos (\omega t+\beta)+12 A_{Z 0}^{2} t \frac{a_{1}}{\omega^{2}}(\sin (\omega t+\beta)-\sin \beta) \\
& -120 A_{Z 0}^{3} t^{3} \frac{a_{3}}{\omega} \cos (\omega t+\beta)+288 A_{Z 0}^{3} t^{2} \frac{a_{3}}{\omega^{2}} \sin (\omega t+\beta) \\
& +432 A_{Z 0}^{3} t \frac{a_{3}}{\omega^{3}} \cos (\omega t+\beta)-144 A_{Z 0}^{3} t \frac{a_{3}}{\omega^{3}} \cos \beta \\
& -288 A_{Z 0}^{3} \frac{a_{3}}{\omega^{4}}(\sin (\omega t+\beta)-\sin \beta) \\
& \left\{\mathrm{x}^{2}\right\}=2 A_{X 0} t \\
& +2 \frac{a_{0}^{2}}{\omega^{2}}\left(\cos \beta-2 \cos (\omega t+\beta) \cos \beta+\cos ^{2}(\omega t+\beta)\right) \\
& +2 A_{Z 0} \frac{a_{0} a_{2}}{\omega^{2}}(t+t \cos 2(\omega t+\beta)-2 t \cos \beta \cdot \cos (\omega t+\beta) \\
& -\frac{\sin 2(\omega t+\beta)-\sin 2 \beta}{\omega} \\
& \left.-2 \sin \beta \frac{\cos \beta-\cos (\omega t+\beta)}{\omega}+2 \cos \beta \frac{\sin (\omega t+\beta)-\sin \beta}{\omega}\right) \\
& +2 A_{\mathrm{ZO}} \frac{a_{1}^{2}}{\omega^{2}}\left(t+\frac{1}{2} t \cos 2(\omega t+\beta)\right. \\
& -\frac{3}{4} \frac{\sin 2(\omega t+\beta)-\sin 2 \beta}{\omega} \\
& \left.-2 \sin \beta \frac{\cos \beta-\cos (\omega t+\beta)}{\omega}\right) \\
& +2 A_{Z 0}^{2} \frac{a_{2}^{2}}{\omega^{2}}\left(5 t^{2}+3 t^{2} \cos 2(\omega t+\beta)-8 t \frac{\sin 2(\omega t+\beta)}{\omega}+4 t \sin \beta \frac{\cos (\omega t+\beta)}{\omega}\right. \\
& -8 \frac{\cos 2(\omega t+\beta)-\cos 2 \beta}{\omega^{2}}+16 \cos \beta \frac{\cos (\omega t+\beta)-\cos \beta}{\omega^{2}} \\
& \left.-4 \sin \beta \frac{\sin (\omega t+\beta)-\sin \beta}{\omega^{2}}\right)
\end{aligned}
$$




$$
\begin{aligned}
& +2 A_{Z 0}^{2} \frac{a_{1} a_{3}}{\omega^{2}}\left(9 t^{2}+6 t^{2} \cos 2(\omega t+\beta)-15 t \frac{\sin 2(\omega t+\beta)-\sin 2 \beta}{\omega}\right. \\
& +12 t \sin \beta \frac{\cos (\omega t+\beta)}{\omega}-\frac{27}{2} \frac{\cos 2(\omega t+\beta)-\cos 2 \beta}{\omega^{2}} \\
& \left.+24 \cos \beta \frac{\cos (\omega t+\beta)-\cos \beta}{\omega^{2}}-12 \sin \beta \frac{\sin (\omega t+\beta)-\sin \beta}{\omega^{2}}\right) \\
& +2 A_{Z 0}^{3} \frac{a_{3}^{2}}{\omega^{2}}\left(48 t^{3}-117 t^{2} \frac{\sin 2(\omega t+\beta)}{\omega}-144 \frac{t}{\omega^{2}}\right. \\
& +30 t^{3} \cos 2(\omega t+\beta)-225 t \frac{\cos 2(\omega t+\beta)}{\omega^{2}} \\
& +144 t \cos \beta \frac{\cos (\omega t+\beta)}{\omega^{2}}+\frac{369}{2} \frac{\sin 2(\omega t+\beta)-\sin 2 \beta}{\omega^{3}} \\
& \left.-288 \sin \beta \frac{\cos (\omega t+\beta)-\cos \beta}{\omega^{3}}-144 \cos \beta \frac{\sin (\omega t+\beta)-\sin \beta}{\omega^{3}}\right) \\
& \begin{aligned}
\sigma_{X}^{2} & =\left\{x^{2}\right\}-\{x\}^{2} \\
& =2 A_{X 0} t
\end{aligned}
\end{aligned}
$$$$
+A_{z 0} \frac{a_{1}^{2}}{\omega^{2}}\left(2 t+t \cos 2(\omega t+\beta)-\frac{3}{2} \frac{\sin 2(\omega t+\beta)-\sin 2 \beta}{\omega}\right.
$$$$
\left.+4 \sin \beta \frac{\cos (\omega t+\beta)-\cos \beta}{\omega}\right)
$$$$
+A_{Z 0}^{2} \frac{a_{2}^{2}}{\omega^{2}}\left(8 t^{2}+4 t^{2} \cos 2(\omega t+\beta)-12 t \frac{\sin 2(\omega t+\beta)}{\omega}\right.
$$$$
\left.-14 \frac{\cos 2(\omega t+\beta)-\cos 2 \beta}{\omega^{2}}+32 \cos \beta \frac{\cos (\omega t+\beta)-\cos \beta}{\omega^{2}}\right)
$$$$
+A_{Z 0}^{2} \frac{a_{1} a_{3}}{\omega^{2}}\left(18 t^{2}+12 t^{2} \cos 2(\omega t+\beta)-30 t \frac{\sin 2(\omega t+\beta)-\sin 2 \beta}{\omega} .\right.
$$$$
+24 t \sin \beta \frac{\cos (\omega t+\beta)}{\omega}-27 \frac{\cos 2(\omega t+\beta)-\cos 2 \beta}{\omega^{2}}
$$$$
\left.+48 \cos \beta \frac{\cos (\omega t+\beta)-\cos \beta}{\omega^{2}}-24 \sin \beta \frac{\sin (\omega t+\beta)-\sin \beta}{\omega^{2}}\right)
$$$$
+A_{Z 0}^{3} \frac{a_{3}^{2}}{\omega^{2}}\left(96 t^{3}+60 t^{3} \cos 2(\omega t+\beta)-234 t^{2} \frac{\sin 2(\omega t+\beta)}{\omega}\right.
$$$$
-288 \frac{t}{\omega^{2}}-450 t \frac{\cos 2(\omega t+\beta)}{\omega^{2}}+288 t \cos \beta \frac{\cos (\omega t+\beta)}{\omega^{2}}
$$$$
+369 \frac{\sin 2(\omega t+\beta)-\sin 2 \beta}{\omega^{3}}-576 \sin \beta \frac{\cos (\omega t+\beta)-\cos \beta}{\omega^{3}}
$$$$
\left.-288 \cos \beta \frac{\sin (\omega t+\beta)-\sin \beta}{\omega^{3}}\right) .
$$ 


\section{Appendix 3}

The Carter and Okubo [1965] model is reviewed briefly in Okubo [1968]. The calculation error appears when the variance is computed from the concentration $A-14((3)$ in Okubo [1968]). The concentration is symmetrical in $y$ and $z$, which means that interchanging $y$ and $z$ and the corresponding exchange coefficients and shear parameters do not change the result. When the operator $\iint_{-\infty}^{\infty} \int_{\infty} x^{2} \mathrm{~d} x \mathrm{~d} y \mathrm{~d} z$, symmetrical in $y$ and $z$ too, is applied on that expression, the resulting expression must also be symmetrical. Carter and Okubo's result for $\sigma_{X}^{2}$ is not, whereas the present author's is.

The second derivation of $A 13$, with respect to $(i k)$ divided by the total mass $M$ and taken at $k=l=m=0$, gives the variance $\sigma_{X}^{2}$.

The result obtained in this way is:

$$
\sigma_{X}^{2}=2 A_{X 0} t+\frac{2}{2}\left(A_{Y 0} b_{1}^{2}+A_{Z 0} a_{1}^{2}\right) t^{3},
$$

which is in agreement with (21).

\section{Appendix 4}

The moments necessary to calculate the variance in the case of the vertical exchange coefficient varying linearly with depth are:

$$
\begin{aligned}
\{z\}= & A_{Z 1} t \\
\left\{z^{2}\right\}= & 2 A_{Z 0} t+2 A_{Z 1}^{2} t^{2} \\
\left\{z^{3}\right\}= & 12 A_{Z 1} A_{Z 0} t^{2}+6 A_{Z 1}^{3} t^{3} \\
\left\{z^{4}\right\}= & 12 A_{Z 0}^{2} t^{2}+72 A_{Z 0} A_{Z 1}^{2} t^{3}+24 A_{Z 1}^{4} t^{4} \\
\left\{z^{5}\right\}= & 180 A_{Z 0}^{2} A_{Z 1} t^{3}+480 A_{Z 0} A_{Z 1}^{3} t^{4}+120 A_{Z 1}^{5} t^{5} \\
\left\{z^{6}\right\}= & 120 A_{Z 0}^{3} t^{3}+2160 A_{Z 0}^{2} A_{Z 1}^{2} t^{4}+3600 A_{Z 0} A_{Z 1}^{4} t^{5}+720 A_{Z 1}^{6} t^{6} \\
\{x\}= & \frac{1}{2} a_{1} A_{Z 1} t^{2}+a_{2} A_{Z 0} t^{2}+\frac{2}{3} a_{2} A_{Z 1}^{2} t^{3}+4 a_{3} A_{Z 0} A_{Z 1} t^{3}+\frac{3}{2} a_{3} A_{Z 1}^{3} t^{4} \\
\{x z\}= & a_{1}\left(A_{Z 0} t^{2}+\frac{5}{6} A_{Z 1}^{2} t^{3}\right)+a_{2}\left(\frac{13}{3} A_{Z 0} A_{Z 1} t^{3}+\frac{5}{3} A_{Z 1}^{3} t^{4}\right) \\
& +a_{3}\left(4 A_{Z 0}^{2} t^{3}+19 A_{Z 0} A_{Z 1}^{2} t^{4}+\frac{51}{10} A_{Z 1}^{4} t^{5}\right) \\
& +a_{3}\left(36 A_{Z 0}^{3} t^{4}+\frac{2733}{5} A_{Z 0}^{2} A_{Z 1}^{2} t^{5}+\frac{3864}{5} A_{Z 0} A_{Z 1}^{4} t^{6}+\frac{4653}{35} A_{Z 1}^{6} t^{7}\right) \\
\left\{x z^{2}\right\}= & a_{1}\left(\frac{17}{3} A_{Z 0} A_{Z 1} t^{3}+\frac{7}{3} A_{Z 1}^{3} t^{4}\right) \\
& +a_{2}\left(\frac{14}{3} A_{Z 0}^{2} t^{3}+\frac{68}{3} A_{Z 0} A_{Z 1}^{2} t^{4}+\frac{92}{15} A_{Z 1}^{4} t^{5}\right) \\
& +a_{3}\left(51 A_{Z 0}^{2} A_{Z 1} t^{4}+\frac{559}{5} A_{Z 0} A_{Z 1}^{3} t^{5}+\frac{117}{5} A_{Z 1}^{5} t^{6}\right) \\
\left\{x Z^{3}\right\}= & a_{1}\left(6 A_{Z 0}^{2} t^{3}+32 A_{Z 0} A_{Z 1}^{2} t^{4}+9 A_{Z 0}^{4} t^{5}\right) \\
& \left.+62 A_{Z 0}^{2} A_{Z 1} t^{4}+\frac{694}{5} A_{Z 0} A_{Z 1}^{3} t^{5}+\frac{146}{5} A_{Z 1}^{5} t^{6}\right) \\
& \\
& \\
\{x &
\end{aligned}
$$




$$
\begin{aligned}
\left\{x^{2}\right\}= & a_{1}^{2}\left(\frac{2}{3} A_{Z 0} t^{3}+\frac{5}{12} A_{Z 1}^{2} t^{4}\right) \\
& +a_{1} a_{2}\left(5 A_{Z 0} A_{Z 1} t^{4}+\frac{8}{5} A_{Z 1}^{3} t^{5}\right) \\
& +a_{1} a_{3}\left(5 A_{Z 0}^{2} t^{4}+\frac{102}{5} A_{Z 0} A_{Z 1}^{2} t^{5}+\frac{47}{10} A_{Z 1}^{4} t^{6}\right) \\
& +a_{2}^{2}\left(\frac{7}{3} A_{Z 0}^{2} t^{4}+\frac{136}{15} A_{Z 0} A_{Z 1}^{2} t^{5}+\frac{184}{90} A_{Z 1}^{4} t^{6}\right) \\
& +a_{2} a_{3}\left(\frac{226}{5} A_{Z 0}^{2} A_{Z 1} t^{5}+\frac{1253}{15} A_{Z 0} A_{Z 1}^{3} t^{6}+\frac{526}{35} A_{Z 1}^{5} t^{7}\right) \\
& +a_{3}^{2}\left(\frac{72}{5} A_{Z 0}^{3} t^{5}+\frac{911}{5} A_{Z 0}^{2} A_{Z 1}^{2} t^{6}+\frac{1104}{5} A_{Z 0} A_{Z 1}^{4} t^{7}+\frac{4653}{140} A_{Z 1}^{6} t^{8}\right) \\
& +2 A_{X 0} t
\end{aligned}
$$

\section{Appendix 5}

The moments necessary to calculate the variances when the vertical exchange coefficient is varying with depth as $z^{2}$ are:

$$
\begin{aligned}
& \left\{z^{2 m+1}\right\}=0 \\
& \left\{z^{4}\right\}=\frac{A_{Z 0}^{2}}{A_{Z 2}^{2}}\left(\frac{3}{35} \mathrm{e}^{20 A Z_{2} t}-\frac{2}{7} \mathrm{e}^{6 A_{Z_{2}} t}+\frac{1}{5}\right) \\
& \left\{z^{6}\right\}=\frac{A_{Z 0}^{3}}{A_{Z 2}^{3}}\left(\frac{5}{231} \mathrm{e}^{42 A_{Z 2} t}-\frac{9}{77} \mathrm{e}^{20 A_{Z 2} t}+\frac{5}{21} \mathrm{e}^{6 A_{Z 2} t}-\frac{1}{7}\right) \\
& \{x\}=\frac{A_{Z 0}}{A_{Z 2}^{2}} a_{2}\left(\frac{1}{18}\left(\mathrm{e}^{6 A_{Z 2} t}-1\right)-\frac{1}{3} A_{Z 2} t\right) \\
& \{x z\}=\frac{A_{Z 0}}{A_{Z 2}^{2}} a_{1}\left(-\frac{1}{4} \mathrm{e}^{2 A_{z 2} t}+\frac{1}{12} \mathrm{e}^{6 A_{Z 2} t}+\frac{1}{6}\right) \\
& +\frac{A_{\mathrm{ZO}}^{2}}{A_{\mathrm{Z} 2}^{3}} a_{3}\left(\frac{1}{210} \mathrm{e}^{20 A_{Z 2} t}-\frac{1}{14} \mathrm{e}^{6 A_{Z 2} t}+\frac{1}{6} \mathrm{e}^{2 A_{Z_{2}} t}-\frac{1}{10}\right) \\
& \left\{x z^{2}\right\}=\frac{A_{Z 0}^{2}}{A_{Z 2}^{3}} a_{2}\left(\frac{3}{490} \mathrm{e}^{20 A_{Z 2} t}-\frac{13}{1323} \mathrm{e}^{6 A_{Z 2} t}-\frac{11}{63} A_{Z 2} t \mathrm{e}^{6 A_{Z 2} t}-\frac{1}{270}+\frac{1}{9} A_{Z 2} t\right) \\
& \left\{x z^{3}\right\}=\frac{A_{Z 0}^{2}}{A_{Z 2}^{3}} a_{1}\left(\frac{3}{280} \mathrm{e}^{20 A_{Z 2} t}-\frac{1}{40} \mathrm{e}^{12 A_{Z 2} t}-\frac{1}{28} \mathrm{e}^{6 A_{Z 2} t}+\frac{3}{20} \mathrm{e}^{2 A_{Z 2} t}-\frac{1}{10}\right) \\
& +\frac{A_{Z 0}^{3}}{A_{Z 2}^{4}} a_{3}\left(\frac{1}{1386} \mathrm{e}^{42 A_{Z 2} t}-\frac{17}{1540} \mathrm{e}^{20 A_{Z 2} t}+\frac{1}{60} \mathrm{e}^{12 A_{Z 2} t}\right. \\
& \left.+\frac{2}{63} \mathrm{e}^{6 A z_{2} t}-\frac{1}{10} \mathrm{e}^{2 A z_{2} t}+\frac{13}{210}\right)
\end{aligned}
$$




$$
\begin{aligned}
\left\{x^{2}\right\}= & \frac{A_{Z 0}}{A_{Z 2}^{3}} a_{1}^{2}\left(\frac{1}{36}\left(\mathrm{e}^{6 A_{Z 2} t}-1\right)-\frac{1}{4}\left(\mathrm{e}^{2 A_{Z 2} t}-1\right)+\frac{1}{3} A_{Z 2} t\right) \\
& +\frac{A_{Z 0}^{2}}{A_{Z 2}^{4}} a_{2}^{2}\left(\frac{3}{4900}\left(\mathrm{e}^{20 A_{Z 2} t}-1\right)+\frac{51}{7938}\left(\mathrm{e}^{6 A_{Z 2} t}-1\right)-\frac{11}{189} A_{Z 2} t \mathrm{e}^{6 A_{Z 2} t}\right. \\
& \left.\quad+\frac{1}{135} A_{Z 2} t+\frac{1}{9} A_{Z 2}^{2} t^{2}\right) \\
& +\frac{A_{Z 0}^{2}}{A_{Z 2}^{4}} a_{1} a_{3}\left(\frac{13}{8400}\left(\mathrm{e}^{20 A_{Z 2} t}-1\right)-\frac{1}{240}\left(\mathrm{e}^{12 A_{Z 2} t}-1\right)-\frac{1}{28}\left(\mathrm{e}^{6 A_{Z 2} t}-1\right)\right. \\
& +\frac{A_{Z 0}^{3}}{A_{Z 2}^{5}} a_{3}^{2}\left(\frac{1}{29106}\left(\mathrm{e}^{42 A_{Z 2} t}-1\right)-\frac{17}{15400}\left(\mathrm{e}^{20 A A_{Z 2} t}-1\right)-\frac{2}{5} A_{Z 2} t\right) \\
& \left.\quad+\frac{1}{360}\left(\mathrm{e}^{12 A_{Z 2} t}-1\right)+\frac{2}{189}\left(\mathrm{e}^{6 A_{Z 2} t}-1\right)-\frac{1}{10}\left(\mathrm{e}^{2 A_{Z 2} t}-1\right)+\frac{13}{105} A_{Z 2} t\right) \\
+ & 2 A_{X 0} t \\
& +\frac{1}{9} A_{X 2} \frac{A_{Z 0}}{A_{Z 2}^{2}}\left(\mathrm{e}^{6 A_{Z 2} t}-1\right)-\frac{2}{3} A_{X 2} \frac{A_{Z 0}}{A_{Z 2}} t .
\end{aligned}
$$

\section{Acknowledgement}

I wish to thank Hjalmar Franz for our many valuable discussions.

\section{References}

Bowden, K.F., 1965: Horizontal mixing in the sea due to a shearing current. J. Fluid Mechanics. 21, 83-95.

Bronstein, I.N. and K.A. Semandjajew, 1979: Taschenbuch der Mathematik. 19. ed. Moscow: Nauka and Leipzig: Teubner, $860 \mathrm{p}$.

Carter, H. and A. Okubo, 1965: A study of the physical processes of movement and dispersion in the Cape Kennedy area. Final report under the U.S. Atomic Energy Commission. Rep. Chesapeake Bay Inst., The Johns Hopkins Univ. No. NYO-2973-1.

Elder, J.W., 1959: The dispersion of marked fluid in turbulent shear flow. J. Fluid Mechanics. 5, 544-560.

Fennel, W., 1979: Theory of turbulent diffusion with arbitrary vertical shear. Beitr. z. Meeresk. No. 42, 17-25.

Franz, H.W., 1982: Turbulenz. 87 p. [Unpubl. manuscript; in German].

Franz, H.W., 1984: Personal communication. [publication in prep.].
Gröbner, W. and N. Hofreiter, 1958: Integraltafel. 2. ed. 2. Teil: Bestimmte Integrale. Wien und Innsbruck: Springer. 204 p.

Hay, J.S. and F. Pasquill, 1959: Diffusion from a continuous source in relation to the spectrum and scale of turbulence. Adv. Geophys. 6, 345365.

Kirwan, A.D., G.J. McNally, E. Reyna and W.J. Merrell, 1978: The near-surface circulation of the Eastern North Pacific. J. phys. Oceanogr. 8, 937-945.

Kullenberg, G., 1971: Vertical diffusion in shallow waters. Tellus, 23, 129-135.

Kullenberg, G., 1972: Apparent horizontal diffusion in stratified vertical shear flow. Tellus. 24, 17-28.

Kullenberg, G., 1974: An experimental and theoretical investigation of the turbulent diffusion in the upper layer of the sea. Københavns Universitet, Institut for Fysisk Oceanografi. Rep. No. 25. 
Mikolajewicz, U., 1984: Betrachtung der Scherdispersion im Meer. Diplomarbeit am Institut für Meereskunde, Universität Hamburg.

Neumann, J., 1978: Some observations on the simple exponential function as a Lagrangian velocity correlation function in turbulent diffusion. Atmosph. Environm. 12, 1965-1968.

Okubo, A., 1962: A review of theoretical models of turbulent diffusion in the sea. Techn. Rep. Chesapeake Bay Inst., The Johns Hopkins Univ. No. 30.

Okubo, A., 1967: The effect of shear in an oscillatory current on horizontal diffusion from an instantaneous source. Int. J. Oceanol. \& Limnol. 1, 194-204.

Okubo, A., 1968: Some remarks on the importance of the "shear effect" on horizontal diffusion. J. Oceanogr. Soc. Japan. 24, 60-69.

Eingegangen am 4. April 1985

Angenommen am 30. September 1985

Anschrift des Verfassers:

Uwe Mikolajewicz, Birkenhof 76, 2390 Flensburg
Okubo, A., 1971: Oceanic diffusion diagrams. Deep-Sea Res. 18, 789-802.

Schott, F. and D. Quadfasel, 1979: Lagrangian and Eulerian measurements of horizontal mixing in the Baltic. Tellus. 31, 138-144.

Taylor, G. I., 1921: Diffusion by continuous movements. Proc. Lond. math. Soc. 20, 196-212.

Taylor, G. I., 1953: Dispersion of soluble matter in solvent flowing slowly through a tube. Proc. Roy. Soc. London (A) 219, 186-203.

Young, W.R., P.B. Rhines and C.J.R. Garret, 1982: Shear-flow dispersion, internal waves and horizontal mixing in the ocean. J. phys. Oceanogr. 12, 515-527. 